Article

\title{
The Use of SMAP-Reflectometry in Science Applications: Calibration and Capabilities
}

\author{
Nereida Rodriguez-Alvarez ${ }^{1, *} \otimes$, Sidharth Misra ${ }^{2}$, Erika Podest ${ }^{3}$, Mary Morris ${ }^{2}($ and \\ Xavier Bosch-Lluis 4 \\ 1 Planetary Radar Radio Science Systems Group, Jet Propulsion Laboratory, California Institute of Technology, \\ Pasadena, CA 91109, USA \\ 2 Microwave Instrument Science Group, Jet Propulsion Laboratory, California Institute of Technology, \\ Pasadena, CA 91109, USA; Sidharth.Misra@jpl.nasa.gov (S.M.); mary.g.morris@jpl.nasa.gov (M.M.) \\ 3 Carbon Cycle and Ecosystems Group, Jet Propulsion Laboratory, California Institute of Technology, \\ Pasadena, CA 91109, USA; Erika.Podest@jpl.nasa.gov \\ 4 Microwave Systems Technology Group, Jet Propulsion Laboratory, California Institute of Technology, \\ Pasadena, CA 91109, USA; javier.bosch-lluis@jpl.nasa.gov \\ * Correspondence: nereida.rodriguez.alvarez@jpl.nasa.gov; Tel.: +1-818-393-7507
}

Received: 31 August 2019; Accepted: 18 October 2019; Published: 21 October 2019

check for updates

\begin{abstract}
The Soil Moisture Active Passive (SMAP) mission became one of the newest spaceborne Global Navigation Satellite System-Reflectometry (GNSS-R) missions collecting Global Positioning System (GPS) bistatic radar measurements when the band-pass center frequency of its radar receiver was switched to the GPS L2C band. SMAP-Reflectometry (SMAP-R) brings a set of unique capabilities, such as polarimetry and improved spatial resolution, that allow for the exploration of scientific applications that other GNSS-R missions cannot address. In order to leverage SMAP-R for scientific applications, a calibration must be performed to account for the characteristics of the SMAP radar receiver and each GPS transmitter. In this study, we analyze the unique characteristics of SMAP-R, as compared to other GNSS-R missions, and present a calibration method for the SMAP-R signals that enables the standardized use of these signals by the scientific community. There are two key calibration parameters that need to be corrected: The first is the GPS transmitted power and GPS antenna gain at the incidence angle of the measured reflections and the second is the convolution of the SMAP high gain antenna pattern and the glistening zone (Earth surface area from where GPS signals scatter). To account for the GPS transmitter variability, GPS instrument properties-transmitted power and antenna gain - are collocated with information collected from the CYclone Global Navigation Satellite System (CYGNSS) at SMAP's range of incidence angles $\left(37.3^{\circ}\right.$ to $\left.42.7^{\circ}\right)$. To account for the convolutional effect of the SMAP antenna gain, both the scattering area of the reflected GPS signal and the SMAP antenna footprint are mapped on the surface. We account for the size of the scattering area corresponding to each delay and Doppler bin of the SMAP-R measurements based off the SMAP antenna pattern, and normalize according to the size of a measurement representative to one obtained with an omnidirectional antenna. We have validated these calibration methods through an analysis of the coherency of the reflected signal over an extensive area of old sea ice having constant surface characteristics over a period of 3 months. By selecting a vicarious scattering surface with high coherency, we eliminated scene variability and complexity in order to avoid scene dependent aliases in the calibration. The calibration method reduced the dependence on the GPS transmitter power and gain from $\sim 1.08 \mathrm{~dB} / \mathrm{dB}$ to a residual error of about $-0.2 \mathrm{~dB} / \mathrm{dB}$. Results also showed that the calibration method eliminates the effect of the high gain antenna filtering effect, thus reducing errors as high as $10 \mathrm{~dB}$ on angles furthest from SMAP's constant $40^{\circ}$ incidence angle.
\end{abstract}

Keywords: GNSS-R; SMAP-Reflectometry; calibration; science applications; high gain antenna 


\section{Introduction}

The Soil Moisture Active Passive (SMAP) mission launched on 31 January, 2015 with the primary objective to provide global measurements of soil moisture and freeze/thaw state every 2-3 days [1]. SMAP carries two scientific instruments, a radiometer and a radar. On 7 July, 2015 the radar stopped working due to an anomaly in the power supply of its high-power amplifier. On 20 August, 2015 the band-pass center frequency of the SMAP radar receiver was switched to $1227.45 \mathrm{MHz}$ enabling the reception of GPS L2C signals as they scatter off the Earth surface. In that moment SMAP became one of the newest Global Navigation Satellite System-Reflectometry (GNSS-R) missions collecting GPS bistatic radar measurements. From here on, the SMAP receiver on reflectometry mode is referred in this manuscript as SMAP-R.

Two other main spaceborne GNSS-R satellite missions have provided sustained measurements for the development of scientific applications. First to space was the Space GPS Receiver Remote Sensing Instrument (SGR-ReSI) on board TechDemoSat-1 (TDS-1) [2]. TDS-1 is an in-orbit test facility that launched on July 2014 with limited lifetime and ceased operations on December 2018. SGR-ReSI has provided GNSS-R measurements from October 2014 to December 2018. Following the success of SGR-ReSI, the CYclone Global Navigation Satellite Systems (CYGNSS) constellation was launched in December 2016 and has been providing measurements since April 2017 [3-5]. Data from these missions enabled a variety of scientific studies. For example, TDS-1 SGR-ReSI measurements have been used to characterize ocean winds [6,7], sea surface height [8], soil moisture and vegetation $[9,10]$, wetland inundation [11,12], sea ice detection and concentration [13-16], sea ice altimetry [17], and sea ice type classification [18]. CYGNSS measurements have been used to observe ocean wind speeds [19-22], soil moisture [23,24], wetlands inundation characterization and dynamics [25-27], and hurricane/tsunami-driven flooding [28,29].

The unique characteristics of the SMAP-R dataset, such as polarimetry, high-gain antenna, and polar coverage, bring new science applications to the GNSS-R field. Figure 1 shows an example of SMAP-R measurement over the ocean.

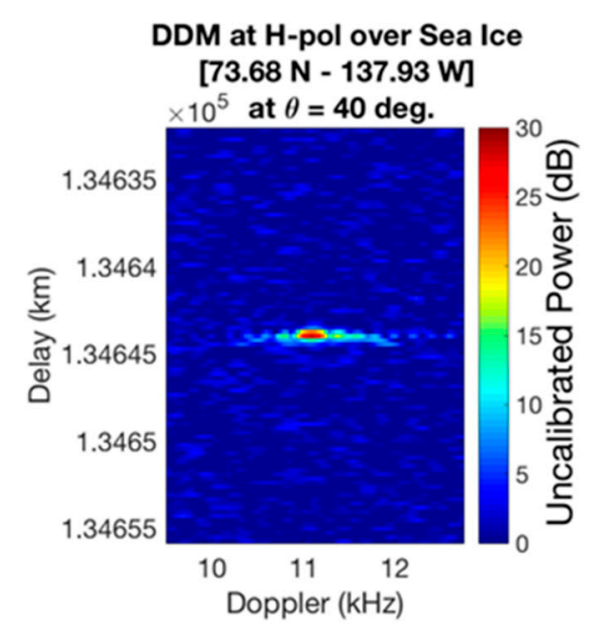

Figure 1. Example of a Soil Moisture Active Passive (SMAP)-Reflectometry (SMAP-R) measurement over the sea ice.

Figure 1 shows the power received by SMAP radar receiver as it scatters from the surface. The measurements are processed into delay and Doppler maps. The spread of the signal and its power is related to the roughness of the surface and its geophysical properties. The smoother a surface is, the more specular the reflection, showing a reduced spread in delay and Doppler. In particular sea ice surface tends to be smooth and present a high reflectivity. The measurements are further explained in Section 3 through mathematical equations. The potential of SMAP-R data has been demonstrated previously in initial assessments of freeze/thaw state identification [30] and polarimetric scatterometry 
over land and cryosphere [31]. Previous work [30,31] assumed that the lack of direct power information had a low impact on the results. Additionally, previous analysis omitted the SMAP antenna filtering effect, which plays an important role in the use of the SMAP-R dataset. Although these initial results ignored the effects due to variations in GPS transmitter power and the SMAP antenna pattern, they helped demonstrate the value of SMAP-R data to the scientific community. Here, we build upon these initial results, presenting work that refines the accuracy of the SMAP-R data, thus providing a better understanding of its performance.

This manuscript presents the methodology developed to produce a SMAP-R calibrated dataset. We analyze the impact of the GPS transmitted power at the incidence angle of the measured reflections and the filtering effect of the SMAP high gain antenna on the measured scattering. Together with the impact analysis, we present a set of methods to mitigate these effects and calibrate the dataset. First, in Section 2 the capabilities of SMAP-R are defined as compared to CYGNSS and TDS-1. Following this comparison, a general method for mapping the variable spatial resolution of each individual GNSS-R measurement is developed. Section 3 details the calibration methodology which accounts for the GPS transmitter variability and the filtering effect of the SMAP high gain antenna. In Section 4 we analyze the impact of this calibration in a SMAP-R dataset characterized by coherent scattering over the Chukchi-Beaufort Sea area covered by mostly old ice in February 2018, with minimal presence of first year ice towards the Alaskan coast. The selected data reduces the variability external to our calibration, allowing us to clearly see the effect of our corrections on the data. In Section 5 we discuss the capabilities and the potential of SMAP-R. Finally, in Section 6 we discuss our conclusions.

\section{Capabilities}

Compared to other GNSS-R datasets currently available from CYGNSS and TDS-1 missions, the SMAP-R dataset offers a number of unique features. We aim to utilize SMAP-R data for estimates of geophysical parameters that are not possible using other GNSS-R missions' data. In the following subsections, we discuss two key aspects that enable different capabilities to sense the Earth surface- the SMAP-R characteristics, as compared to other GNSS-R missions, and the spatial resolution of SMAP-R measurements.

\subsection{SMAP-R Characteristics}

SMAP was not designed to operate as a reflectometer measuring GPS signals. Consequently, some of the characteristics of SMAP-R, such as its poor spatial sampling, are not ideal for some science applications. However, some characteristics, such as the dual-polarization and the high gain antenna, provide an opportunity to study the potential benefits of these unique features. In addition, SMAP provides global measurements, which are important for cryosphere investigations. SMAP-R characteristics include:

1. Low temporal coverage and low sampling limits utility in applications that require high temporal repetition. However, applications that do not require frequently updated information are suitable for SMAP-R. For example, SMAP-R data are suitable for vegetation water content estimation, since these observables are less variable on sub-seasonal timescales (i.e., 15 days -1 month). Figure 2 provides an example of the coverage obtained for 1 day, 15 days, 1 month, and 2 months of data. Figure 2 illustrates that sampling over 1 day is scarce, but after 1 month, the accumulated dataset is suitable for spatial analysis at regional scales. In order to leverage this unique, yet sparse dataset, data must be aggregated over time. After an accumulation of measurements over long periods with low variation, we can define two extreme states as references from which we can investigate transitional periods in time between these two states. These data synthesis techniques have been implemented in [32] for freeze and thaw seasonal transitions in Alaska and in [33] for vegetation water content estimation on the US Corn Belt agricultural area. The technique allows for an understanding of how the observed surfaces transition from one state to another, for example, from freeze and thaw states [32], or from bare soil to peak vegetation growth [33]. 
2. SMAP-R provides measurements at vertical (V-) and horizontal (H-) polarizations. Polarimetric information is utilized with the calculation of a polarimetric ratio, which enables the estimation of vegetation water content and crop characteristics. Other applications include freeze/thaw state characterization and sea ice type classification; both freeze/thaw states and different ice types have polarization dependent signatures. Additionally, studies of surface roughness in arid/semi-arid regions (very low vegetation) are possible because roughness also has clear polarimetric components. Polarimetry is also a key element in oil spill detection, but SMAP-R may have limited utility in this application as the poor spatial sampling may not be able to capture these events.

3. SMAP-R has a higher gain antenna than other GNSS-R missions ( $36 \mathrm{~dB}$ compared to the CYGNSS down-looking gain $\sim 14.5 \mathrm{~dB}$ ), allowing for better signal-to-noise ratio (SNR) at shorter integration times. Shorter integration times translate into better along-track spatial resolution for each specular reflection measured. The along-track spatial resolution is the result of the scattering area being elongated by the distance SMAP moved during the integration time. Better SNR leads therefore to improved spatial resolutions.

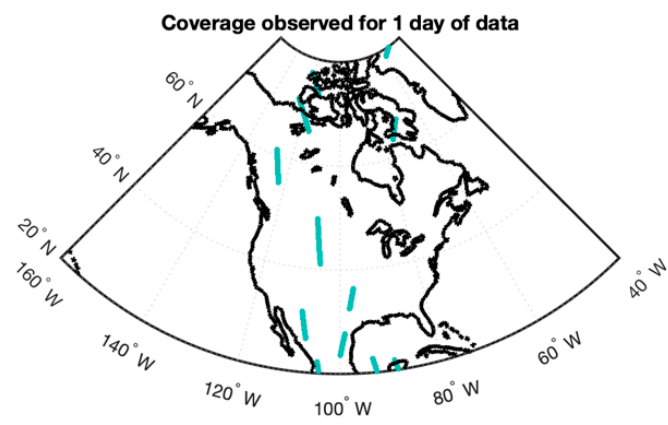

(a)

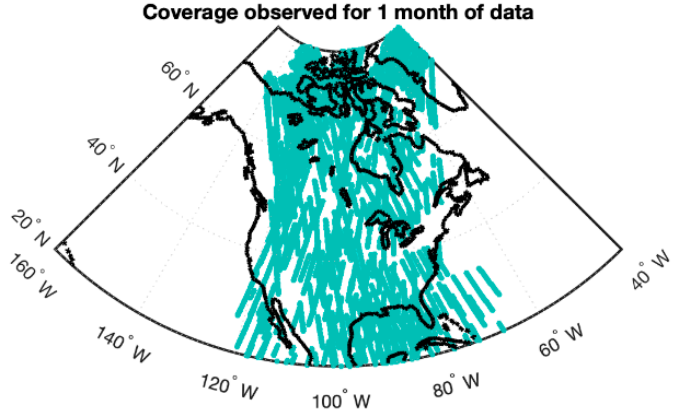

(c)

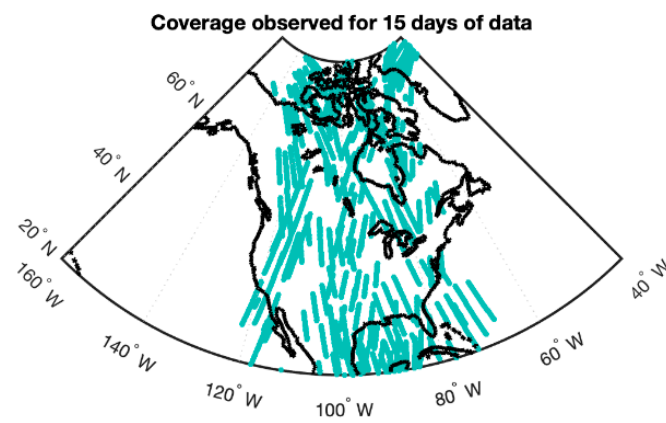

(b)

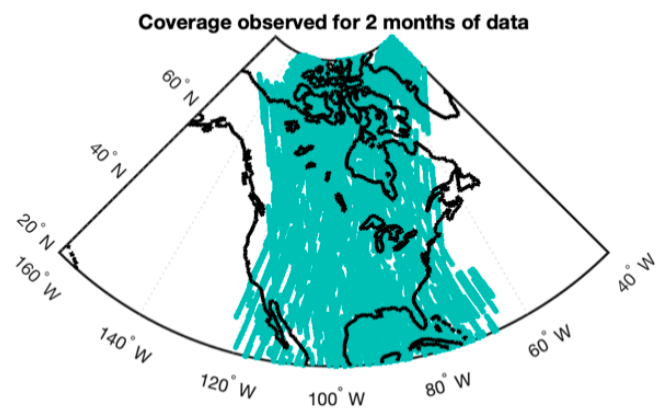

(d)

Figure 2. SMAP-R coverage observed for (a) 1 day (285 observations), (b) 15 days (7168 observations), (c) 1 month (14,449 observations), and (d) 2 months (30,500 observations).

In the next two subsections we specifically compare SMAP-R to the other two GNSS-R missions, CYGNSS and TDS-1. The differences shown are derived from each mission's design requirements and/or in the case of SMAP-R, the repurposing of a mission to use its radar receiver for something it was not originally intended to do.

\subsubsection{SMAP-R vs. CYGNSS}

First, we will compare the characteristics of SMAP-R to CYGNSS. It is useful to compare the spatial and temporal sampling from each platform. Figure 3 shows spatial distribution of the peak SNR of the GPS reflections captured by SMAP-R and CYGNSS over one day.

Figure 3 clearly shows that the sampling from SMAP over one day is limited when compared to CYGNSS, the sampling from SMAP over one day is limited. Tracks measured by SMAP-R are shorter in length in part because its beamwidth limits the field of view and incidence angles to a small range of $37.3^{\circ}$ to $42.7^{\circ}$. Additionally, since the antenna rotates at $14.6 \mathrm{rpm}$, there are gaps of 
$\sim 25 \mathrm{~km}$ between SMAP-R observations along each track, one observation period every $4 \mathrm{~s}$. There are some benefits to the high gain antenna. Notably, Figure 3 shows the SNR range obtained for SMAP-R (30 dB for $25 \mathrm{~ms}$ integration time) and the SNR range obtained from CYGNSS ( $25 \mathrm{~dB}$ for $1000 \mathrm{~ms}$ integration time). The two platforms observe similar ranges of SNR, but SMAP-R data are processed at shorter integrations times. Integration time impacts the spatial resolution of each measurement. For surfaces with dominant coherence reflections (e.g., smooth surfaces), such as lakes and/or sea ice, the collected scattered energy comes from an area of about $800 \mathrm{~m} \times 1 \mathrm{~km}$. SMAP-R integrates the received signal over $25 \mathrm{~ms}$, smearing the observation $180 \mathrm{~m}$ in the along-track direction. On the other hand, CYGNSS integrates the received signal over $1000 \mathrm{~ms}$, elongating the observation $6 \mathrm{~km}$ in the along-track direction. Consequently, the spatial resolution possible from SMAP-R observations is better than that of CYGNSS for similar SNR.

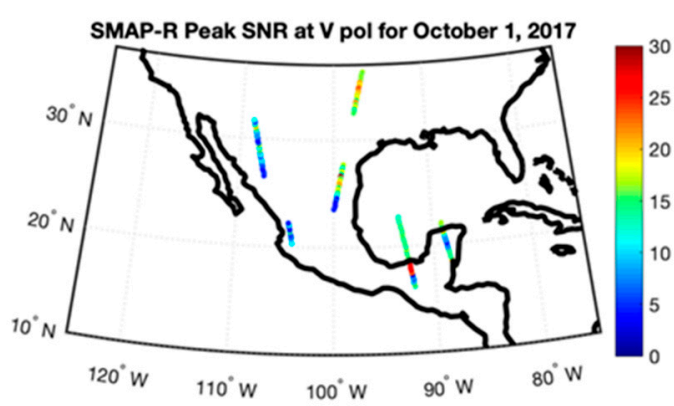

(a)

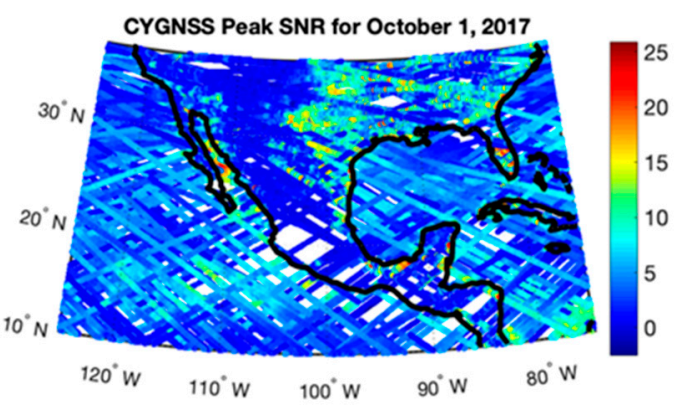

(b)

Figure 3. Peak signal to noise ratio (SNR) of the GPS reflections, captured by (a) SMAP-R at V-polarization with $25 \mathrm{~ms}$ integration time and (b) CYGNSS, for 1 day of measurements with 1,000 ms integration time. Peak SNR plotted in dB.

SMAP and CYGNSS are in different orbits, and therefore cover different latitudinal ranges. CYGNSS covers only the tropics, while SMAP-R provides global coverage, including the poles. SMAP-R therefore allows for studies of such sea ice characterization in the poles (both Antarctic and Arctic) or freeze/thaw analysis.

Finally, the frequency and polarization are different between instruments. CYGNSS receives at GPS L1 band (frequency $=1575.42 \mathrm{MHz}$, wavelength $=19 \mathrm{~cm}$ ) while SMAP-R receives at GPS L2C band (frequency $=1227.45 \mathrm{MHz}$, wavelength $=24.42 \mathrm{~cm}$ ). CYGNSS measures in left-hand circularly polarization (LHCP) while SMAP measures in V-polarization and H-polarization.

\subsubsection{SMAP-R vs. TDS-1(SGR-ReSI)}

TDS-1, with SGR-ReSI—an instrument specifically designed to perform as a reflectometer-onboard also serves as a useful comparison. SGR-ReSI receives at GPS L1 band (frequency $=1575.42 \mathrm{MHz}$, wavelength $=19 \mathrm{~cm}$ ) while SMAP-R receives at GPS L2C band (frequency $=1227.45 \mathrm{MHz}$, wavelength $=24.42 \mathrm{~cm}$ ). TDS-1 measures in LHCP while SMAP measures in V-polarization and H-polarization. Figure 4 shows an example of the sampling for the GPS signals captured by SMAP-R and TDS- 1 for 1 day of measurements.

Figure 4 shows that TDS- 1 coverage is greater than that from SMAP-R. However, as previously shown in Figure 3, the differences in coverage between SMAP-R and TDS-1 are smaller in comparison with the differences between SMAP-R and CYGNSS.

The observed SNR range for TDS- 1 is also $30 \mathrm{~dB}$. While TDS-1 orbits at a slightly different speed and altitude than CYGNSS, TDS-1 integrates the received signal similarly over $1000 \mathrm{~ms}$, creating a smeared observation over $7.6 \mathrm{~km}$ in the along-track direction. The spatial resolution attained from SMAP-R samples is therefore also better than that of the TDS-1 mission.

Regardless of the instrument, spatial patterns of observed reflections are variable, with no exact repetition. Sampling depends on GPS constellation geometries coinciding within the receiver field of 
view. Even though the sampling patterns of reflections are pseudo random in nature, SMAP-R data are collected continuously. On the contrary, the operation time of SGR-ReSI (onboard TDS-1) was limited to two out of eight days. Data collection from SGR-ReSI was limited because TDS-1 was an on-orbit test facility, so measurement time was shared between SGR-ReSI and other instruments.

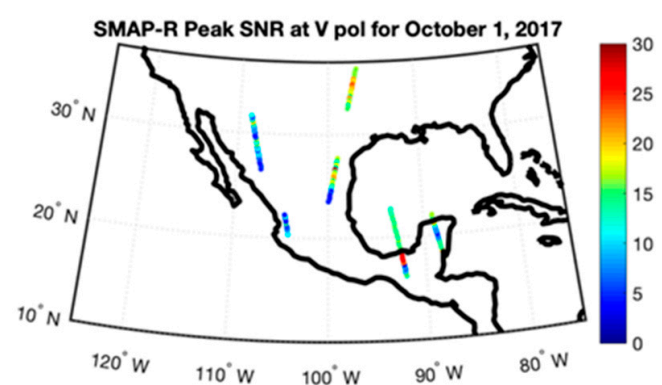

(a)

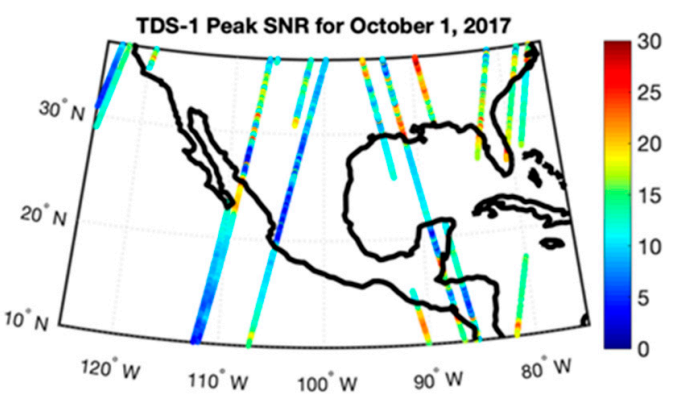

(b)

Figure 4. Peak signal to noise ratio (SNR) of the GPS reflections captured by (a) SMAP-R at V-polarization 25 ms integration time and (b) TDS-1 for 1 day of measurements with 1000 ms integration time. Peak SNR plotted in $\mathrm{dB}$.

\subsection{SMAP-R Spatial Resolution}

In order to determine the capabilities of SMAP-R for science applications, there is a need to determine the footprint of the SMAP-R measurements. The spatial resolution of GNSS-R observations is variable and depends on the scattering surface, the incidence angle, and the integration time. The following methodology applies to SMAP-R, CYGNSS, and TDS-1 measurements, regardless of the scattering surface and incidence angle. The measurement obtained from a GNSS-R receiver is a delay-Doppler map (DDM), [34-36] which represents the power scattering of the GPS signal over the Earth surface collected at different values of delay and Doppler. We present a general approach to map the DDM information to its native spatial resolution [37-40]:

1. A power threshold is applied to the DDM in order to estimate the area from where the scattering signal is coming from. In order to set a threshold, most of the area representing the total power needs to be accounted for. We select a threshold that gathers the $\mathrm{N} \%$ of the total power, $\mathrm{N}$ being an arbitrary number. $\mathrm{N} \%$ in our studies has been selected to at least $80 \%$. This threshold defines a delay and a Doppler value for the area under consideration.

2. Map the delay and Doppler values into the surface, drawing the iso-delay and Doppler lines. Both delay and Doppler provide the exact area of the scattering considered. For the sake of simplicity, we use the delay value alone, which translates to an ellipse in the surface.

3. The ellipse is centered in the specular point and is rotated to the scattering plane formed by the receiver and transmitter geometries, with the semi-major ellipse axis aligned with the scattering plane and the semi-minor axis perpendicular to it.

4. The ellipse is then elongated by the distance obtained by multiplying the integration time by the velocity of the satellite in the along-track direction.

5. The final elongated ellipse is mapped into the surface, with a fine enough grid that allows the delineation of the shape of such ellipse.

6. Steps 1 to 5 are repeated for all measurements.

We have applied the described methodology to SMAP-R measurements (Table 1). Similar studies could be done for the other GNSS-R missions (TDS-1 and CYGNSS). The methodology is therefore general, but results are mission-dependent. Table 1 shows the mean and the standard deviation of the size of the scattering area computed for a number of scenes and $\mathrm{N} \%$ threshold values. Explored scenarios are sea ice, lakes, wetlands, arid land, low vegetation, high vegetation, and ocean. The $\mathrm{N}$ value ranges from $80 \%$ to $60 \%$, in steps of $5 \%$. When a threshold $\mathrm{N}=80 \%$ is used, the DDM signals 
from land, vegetated, and ocean scenes, overflow to the system noise instead of staying in the actual scattering power, which erroneously increases the value the mean and standard deviation.

Table 1. Mean size of the scattering area (diameter) of GPS reflections over a variety of scenes and N values as measured by SMAP-R.

\begin{tabular}{|c|c|c|c|c|c|c|c|c|c|c|}
\hline \multirow{2}{*}{ Scenario } & \multicolumn{2}{|c|}{$\mathrm{N}=80 \%$} & \multicolumn{2}{|c|}{$\mathrm{N}=75 \%$} & \multicolumn{2}{|c|}{$\mathrm{N}=70 \%$} & \multicolumn{2}{|c|}{$\mathrm{N}=65 \%$} & \multicolumn{2}{|c|}{$\mathrm{N}=60 \%$} \\
\hline & $\mu(\mathbf{k m})$ & $\sigma(\mathbf{k m})$ & $\mu(\mathbf{k m})$ & $\sigma(\mathbf{k m})$ & $\mu(\mathrm{km})$ & $\sigma(\mathrm{km})$ & $\mu(\mathbf{k m})$ & $\sigma(\mathbf{k m})$ & $\mu(\mathbf{k m})$ & $\sigma(\mathbf{k m})$ \\
\hline Sea Ice & 2.8 & 0.9 & 2.4 & 0.8 & 2.1 & 0.6 & 1.8 & 0.3 & 1.8 & 0.25 \\
\hline Lake & 2.7 & 0.8 & 2.6 & 0.9 & 2.3 & 0.7 & 1.5 & 0.25 & 1.5 & 0.26 \\
\hline Wetland & 9.6 & 2.8 & 3.3 & 1.4 & 2.8 & 0.5 & 2.4 & 0.34 & 2.1 & 0.28 \\
\hline Arid Land & 13.3 & 5.8 & 6.4 & 4.2 & 5.3 & 2.6 & 4.9 & 2.3 & 4.3 & 2.4 \\
\hline Low Vegetation & 20.2 & 6.6 & 12.5 & 5.6 & 12.1 & 4.2 & 11.9 & 3.9 & 8.6 & 3.3 \\
\hline High Vegetation & 43.1 & 7.3 & 38.9 & 6.5 & 26.6 & 4.5 & 26.2 & 4.1 & 23.5 & 8.5 \\
\hline Ocean & 45.3 & 8.4 & 44.8 & 7.9 & 44.6 & 7.6 & 42.3 & 6.8 & 41.1 & 6.4 \\
\hline
\end{tabular}

The thresholds $\mathrm{N}=65 \%$ and $\mathrm{N}=60 \%$ are not accounting for all the scattering power from the scene, missing important contribution and hence the threshold tends to underestimate the size of the footprint. The range within $\mathrm{N}=70 \%$ and $\mathrm{N}=75 \%$ show all the scenarios with similar stable behavior. For $\mathrm{N}=75 \%$ we observe an averaged spatial resolution of $2.4 \mathrm{~km}$ for sea ice scenarios. A spatial resolution of $2.6 \mathrm{~km}$ is observed for the lakes and $3.3 \mathrm{~km}$ for wetlands, even in cases where there is vegetation. The spatial resolution decreases over arid land with $6.4 \mathrm{~km}$ and is even lower when there is vegetation, starting at $12.5 \mathrm{~km}$ for low vegetation volume and up to $38.9 \mathrm{~km}$ on high vegetation areas. Notice that when $\mathrm{N}=75 \%$ for high vegetation scenes, the DDM noise might still prevail due to the lower power of the high vegetation scenes. The true spatial resolution may be found when $\mathrm{N}=70 \%$, giving an averaged spatial resolution of a circle $26.6 \mathrm{~km}$ diameter. Finally, the coarser resolution is obtained over ocean, where $44.8 \mathrm{~km}$ diameter resolution is achieved. For an ocean scenario, the size of the scattering zone can expand over hundreds of kilometers depending on its roughness, i.e., wind speed, but SMAP-R is observing the scattering power filtered by its antenna. The SMAP antenna's $-3 \mathrm{~dB}$ beamwidth footprint is estimated to be $40 \mathrm{~km}$, therefore SMAP measures scattering power from slightly further away from its antenna's $-3 \mathrm{~dB}$ beamwidth.

The incidence angle of the GPS reflections has not been accounted for in Table 1, but it is an important parameter to consider. Derived from an analysis similar to the one shown in Table 1, a look-up-table (LUT) linking the size of the scattering area (semi-major and semi-minor axis of an ellipse) to the delay that accounts for the $\mathrm{N} \%$ of the total scattering observed with the incidence angle can be computed. This LUT would be used reduce the computational cost of the calculation of iso-delay and iso-Doppler lines for each measurement. In other words, the DDM provides the delay that represents $\mathrm{N} \%$ of the total power observed as well as the incidence angle of the measurement. From these two variables, the LUT provides the size of an ellipse representative of the scattering area. The LUT approach is not surface-dependent, and therefore is valid for any type of class such as sea ice, wetlands, rivers, lakes, bare soils, vegetated soils, desserts, and oceans. Sea ice DDMs contain the $\mathrm{N} \%$ of the power within short delays, while ocean DDMs contain the $\mathrm{N} \%$ of the power within longer delays. Future studies will include analyzing the selection of $\mathrm{N}$ to be representative of the scattering area accounting for the incidence angle. Another approach might include selecting the DDM samples over the noise level for each DDM instead of a fixed N\% value.

We also need to consider how the SMAP antenna pattern will constrain the DDM footprint in some instances (e.g., oceans or highly vegetated areas). Further discussion of the impact of the SMAP antenna pattern on the DDM footprint is discussed in Section 3.2.

\section{Calibration of SMAP-R Signals}

The mathematical equations that describe the collected power over a scattering surface of the GPS signal (i.e., DDM) were defined in $[35,36]$ and can easily be found in the literature. Those equations are 
presented here to illustrate the SMAP-R differences with respect to other missions. Equation (1) describes the reflected GPS signal that would be received from a surface dominated by incoherent scattering.

$$
P_{\text {incoh }}\left(\tau, f_{d}\right)=T_{i}^{2} \int \frac{P_{t x} G_{t x} G_{r x}(\vec{\rho}) \lambda^{2} \Lambda^{2}\left(\tau-\tau_{s}(\vec{\rho})\right)}{(4 \pi)^{3} R_{r x}^{2}(\vec{\rho}) R_{t x}^{2}(\vec{\rho})} \times\left|S\left(f_{d}(\vec{\rho})-f\right)\right|^{2} \sigma_{0}(\vec{\rho}) d^{2} \rho
$$

where

- $\quad \vec{\rho}$ defines the different surface pixels within the scattering area;

- $\mathrm{T}_{\mathrm{i}}$ is the coherent integration time;

- $P_{\mathrm{tx}}$ is the GPS transmitted power;

- $\mathrm{G}_{\mathrm{tx}}$ is the GPS transmitter antenna gain;

- $\mathrm{G}_{\mathrm{rx}}$ is the receiver antenna gain;

- $\lambda$ is the GPS signal wavelength (at GPS-L2C is $24.42 \mathrm{~cm}$ );

- $\mathrm{R}_{\mathrm{tx}}$ corresponds to the distance from the transmitter to a particular surface pixel $(\vec{\rho})$;

- $\mathrm{R}_{\mathrm{rx}}$ corresponds to the distance from the receiver to a particular surface pixel $(\vec{\rho})$;

- $\quad$ The sinc (S) function defines the spread of the signal in Doppler;

- The delta $\Lambda$ function defines the spread of the signal in time;

- $\sigma_{0}(\vec{\rho})$ is the bistatic radar cross-section of a rough surface and is defined as:

- $\sigma_{0}(\vec{\rho})=\pi|\mathcal{R}(\vec{\rho})|^{2} \frac{\mathrm{q}^{4}(\vec{\rho})}{\mathrm{q}_{\mathrm{z}}^{4}(\vec{\rho})} \wp\left(-\frac{\mathrm{q}_{\perp}(\vec{\rho})}{\mathrm{q}_{\mathrm{z}}(\vec{\rho})}\right)$,

where $\mathcal{R}$ is the Fresnel reflection coefficient, $\wp$ is the probability density function of the surface slopes and $\mathrm{q}_{\mathrm{z}}$ and $\mathrm{q}_{\perp}$ are the tangential and perpendicular components of the bisector vector or scattering vector $\overrightarrow{\mathrm{q}}$.

Equation (2) describes the reflected GPS signal that would be received from a surface dominated by coherent scattering $[15,36]$.

$$
P_{\text {coh }}\left(\tau, \mathrm{f}_{\mathrm{d}}\right)=\mathrm{T}_{\mathrm{i}}^{2} \frac{\mathrm{P}_{\mathrm{tx}} \mathrm{G}_{\mathrm{tx}} \mathrm{G}_{\mathrm{rx}}\left(\vec{\rho}_{\mathrm{sp}}\right) \lambda^{2} \Lambda^{2}\left(\tau-\tau_{\mathrm{s}}\left(\vec{\rho}_{\mathrm{sp}}\right)\right)}{(4 \pi)^{3}\left(\mathrm{R}_{\mathrm{rx}}\left(\vec{\rho}_{\mathrm{sp}}\right)+\mathrm{R}_{\mathrm{tx}}\left(\vec{\rho}_{\mathrm{sp}}\right)\right)^{2}} \times\left|\mathrm{S}\left(\mathrm{f}_{\mathrm{d}}\left(\vec{\rho}_{\mathrm{sp}}\right)-\mathrm{f}\right)\right|^{2}\left|\overline{\mathcal{R}}\left(\vec{\rho}_{\mathrm{sp}}\right)\right|^{2}
$$

where:

- $\quad \vec{\rho}_{\text {sp }}$ corresponds to the specular point on the surface;

- $\overline{\mathcal{R}}\left(\vec{\rho}_{\mathrm{sp}}\right)$ is an average reflection coefficient at the specular point, obtained from the average reflection coefficient of the scattering surface $\overline{\mathcal{R}}(\vec{\rho})$ evaluated at the specular direction, i.e., $\overline{\mathcal{R}}\left(\vec{\rho}_{\mathrm{sp}}\right)=\overline{\mathcal{R}}(\vec{\rho}) \delta\left(\vec{\rho}-\vec{\rho}_{\mathrm{sp}}\right)$.

SMAP-R data presents calibration challenges since the GPS direct power $\left(\mathrm{P}_{\mathrm{tx}}\right)$ is not measured and there is a lack of knowledge about GPS antenna gain $\left(G_{t x}\right)$. In addition, the SMAP high antenna gain results in a footprint of $40 \mathrm{~km}$ diameter at a $40^{\circ}$ incidence angle that limits the number of specular bistatic observations. Therefore, to obtain $\sigma_{0}(\vec{\rho})$ or $\overline{\mathcal{R}}\left(\vec{\rho}_{\text {sp }}\right)$ from Equations (1) and (2), there are a number of calibration corrections that need to be applied to the received power in watts $\left(\mathrm{P}_{\text {incoh }}\right.$ or $\mathrm{P}_{\text {coh }}$, depending on the type of scattering surface). The next two subsections provide the calibration methodology, which uses external information from CYGNSS to account for the direct Equivalent Radiated Isotropically Power (EIRP) information $\left(\mathrm{P}_{\mathrm{tx}} \mathrm{G}_{\mathrm{tx}}\right)$ and the calibration methodology to account for the filtering effect of the SMAP antenna. The two calibration methodologies are analyzed under two different scenarios:

1. A surface dominated by incoherent scattering, i.e., Equation (1), either the ocean surface or land areas either covered or not by vegetation with no presence of river, lakes, wetlands, flooding, or any kind of coherently dominated reflection surface; 
2. A surface dominated by coherent scattering, i.e., Equation (2), any type of land area containing mirror-like surfaces (such as rivers, lakes, wetlands, flooded surfaces, or sea ice surfaces).

Note that this work employs CYGNSS version 2.1 data. EIRP values provided in version 2.1 are estimated coarsely and contain errors. Those inaccuracies are a source of error in the calibration depicted in this study. New CYGNSS version 3.0 data will provide more accurate EIRP information.

\subsection{Direct Power Information}

Regardless of the scattering regime-incoherent or coherent-it is important to have knowledge of the transmit power. In both Equations (1) and (2), the direct power information corresponds to both GPS transmitted power $P_{t x}$ and GPS antenna gain in the direction of the specular reflection $G_{t x}$.

In previous publications [30], two assumptions were made:

- $G_{t x}$ is constant due to the fixed observing geometry;

- $\quad P_{t x}$ biases between GPS satellites are small and no greater than $1.8 \mathrm{~dB}$.

In [30], a first assessment of the variability of the freeze/thaw observations used these rough assumptions were considered to have a low impact in the results. To validate the assumptions, we looked into the GPS transmitted power and GPS antenna gain information contained in the metadata files of CYGNSS dataset, shown in Figure 5.

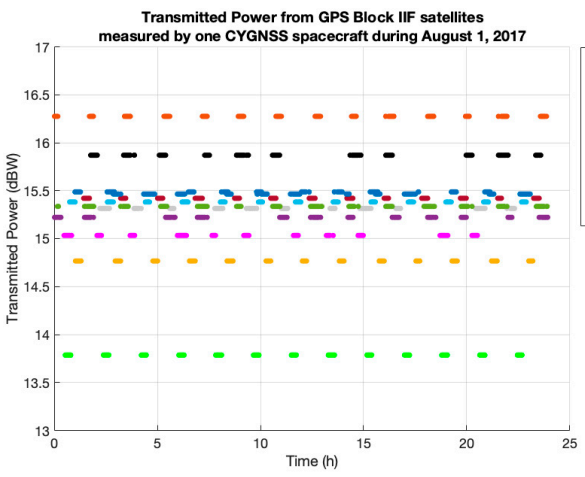

(a)

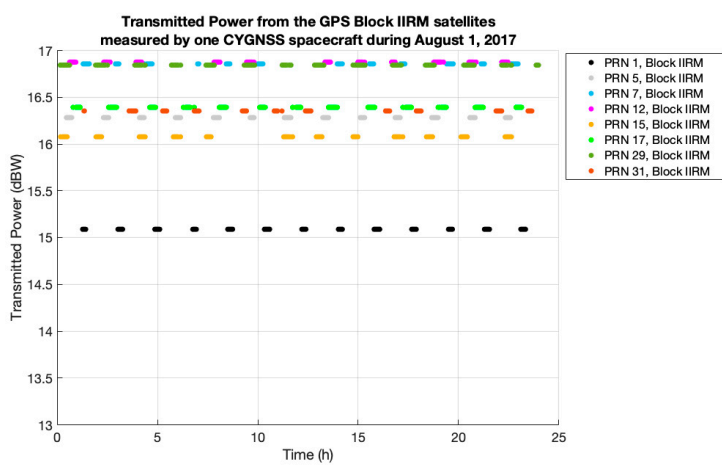

(b)

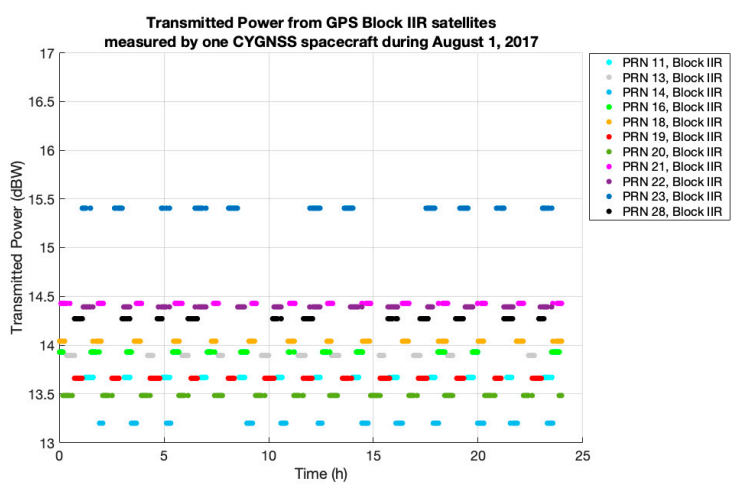

(c)

Figure 5. GPS transmitted power $P_{t x}$ for the 32 satellites of the constellation. The information is in the variation range of those transmitted power. Each color corresponds to a different GPS satellite. (a) GPS block IIF satellites are represented by circles, (b) GPS block IIRM satellites are represented by squares, and (c) GPS block IIR are represented by crosses.

Figure 5 shows that the transmitted power $P_{t x}$ variation between GPS satellites can be up to $3.6 \mathrm{~dB}$, twice as large as the maximum estimated in the first studies [30]. Different GPS blocks cause different 
transmitter power levels. In Figure 5, we have included the legend with information on the type of block: Figure 5a, GPS block IIF satellites; Figure 5b, GPS block IIRM satellites; and Figure 5c, GPS block IIR. Overall, GPS Block IIRM satellites appear to transmit more power as compared to GPS block IIR satellites. GPS block IIF satellites present powers that vary between 13.8 and $16.3 \mathrm{~dB}$. GPS block IIRM and GPS block IIF satellites have the capability to redistribute transmit power between individual signal components (flex power) which is used for protection against jamming [41]. In addition, it has been reported that the transmitter power has a geographical dependence [41] with an impact up to $2.5 \mathrm{~dB}$ on the transmitted power. We use a daily mean of CYGNSS-reported transmitted values, if the power is increased in the middle of the day up to $2.5 \mathrm{~dB}$, we could be introducing a $1.25 \mathrm{~dB}$ error to our calibration. As we rely on CYGNSS, all transmitted power variations included in the dataset would be accounted for in our calibration as daily means, but to increase the accuracy, future work should include a collocation of the transmitted power for the same GPS satellite within minutes.

On top of the transmitted power, the GPS antenna gain $G_{t x}$, which is variable and dependent on the particular GPS space vehicle, should also be considered. Figure 5 shows the antenna gain $G_{t x}$ variability for several GPS satellites for an incidence angle range from $0^{\circ}$ to $72^{\circ}$.

For SMAP-R, only the range of incidence angles of the specular reflections in the SMAP antenna pattern range $37.3^{\circ}$ to $42.7^{\circ}$ needs to be considered. The antenna gain difference observed for all GPS satellites within this range of incidence angles varies from 0.4 to $1 \mathrm{~dB}$. Therefore, by limiting the GPS transmitter values within the range of incidence angles captured by the SMAP antenna, and combining both $P_{t x}$ and $G_{t x}$, i.e., Figures 5 and 6, we calculate the total impact that these variations can have on the received power, shown in Figure 7.

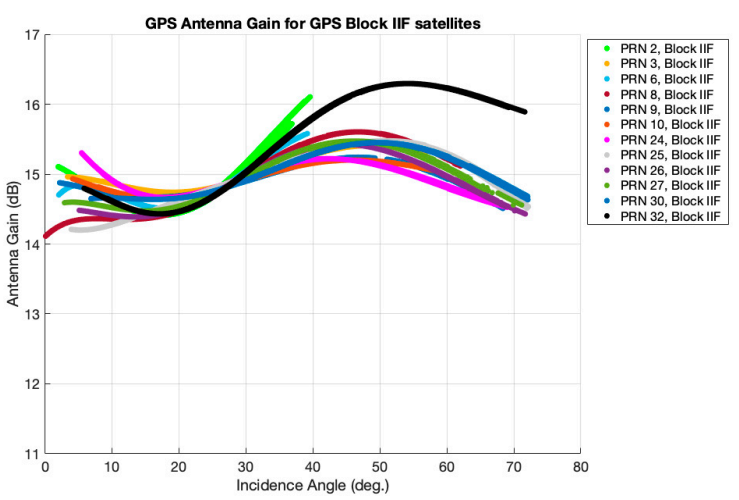

(a)

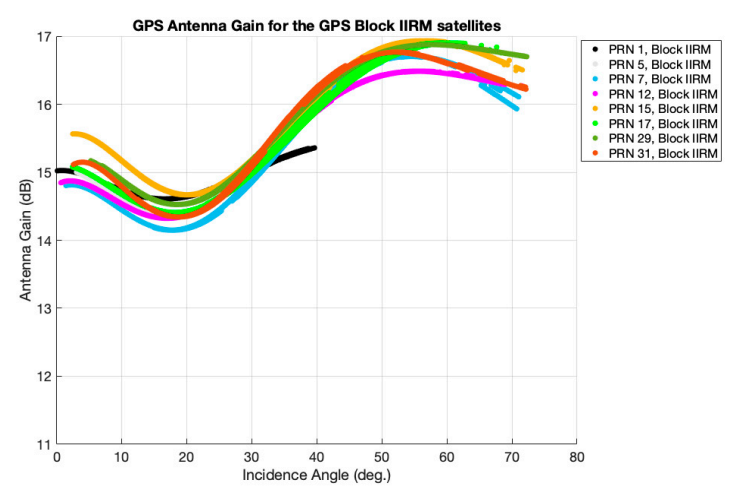

(b)

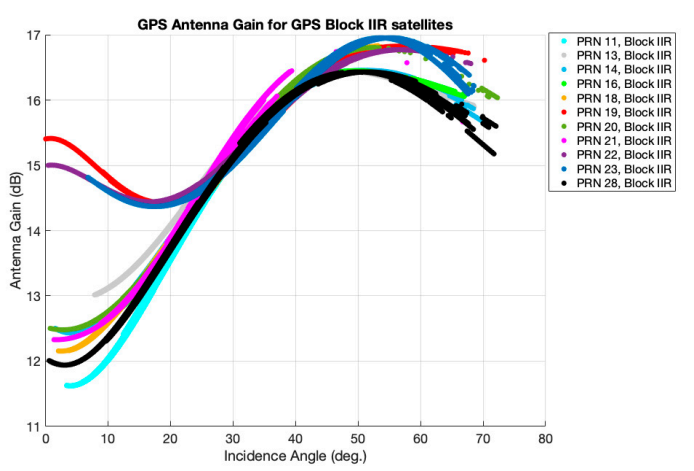

(c)

Figure 6. GPS antenna gain $G_{t x}$ for the 32 satellites of the constellation. The information is in the variation range of those transmitted power. Each color corresponds to a different GPS satellite. Each color corresponds to a different GPS satellite. (a) GPS block IIF satellites are represented by circles, (b) GPS block IIRM satellites are represented by squares, and (c) GPS block IIR are represented by crosses. 
Figure 7 quantifies a total observed difference of $4.2 \mathrm{~dB}$ difference is observed for the combined effect of the GPS EIRP $P_{t x} G_{t x}$ at the specular incidence angle for the range of incidence angles measured by SMAP, i.e., $37.3^{\circ}$ to $42.7^{\circ}$. A difference of $4.2 \mathrm{~dB}$ is not negligible and requires standardization.

GPS transmitter differences must be accounted for using the data for scientific research. Often, the sensitivity of forward scattered signals to geophysical parameters is small. For instance, the dynamic range of freeze/thaw states is comprised within a $4-7 \mathrm{~dB}$ range, depending on the landscape [32]. Proper calibration is also important for applications related to vegetation water content estimation; the variability caused by changes of $2-3 \mathrm{~kg} / \mathrm{m}^{2}$ in vegetation water content corresponds to a change of approximately $5 \mathrm{~dB}$ in the reflected GPS signal [33]. These geophysical sensitivities are within the same order of magnitude than the GPS transmitter variations alone. Therefore, GPS transmitter variations must be accounted for before science research is undertaken.

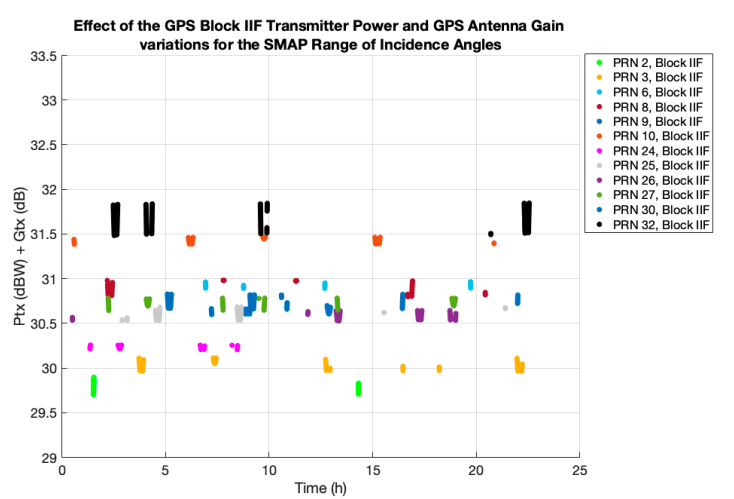

(a)

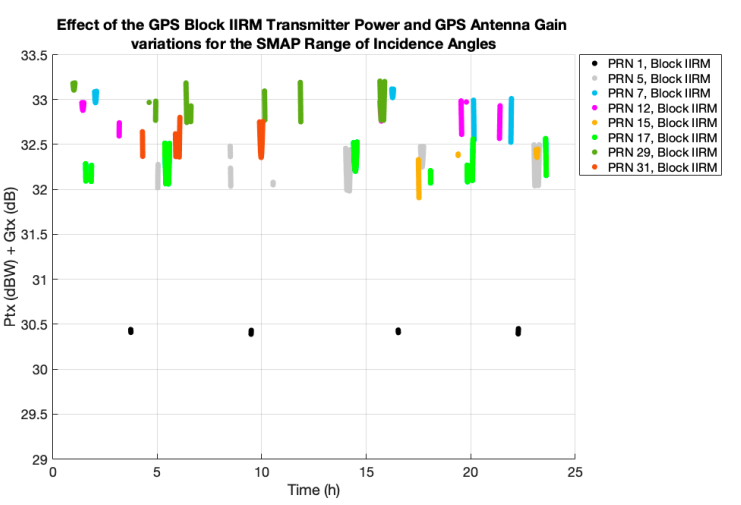

(b)

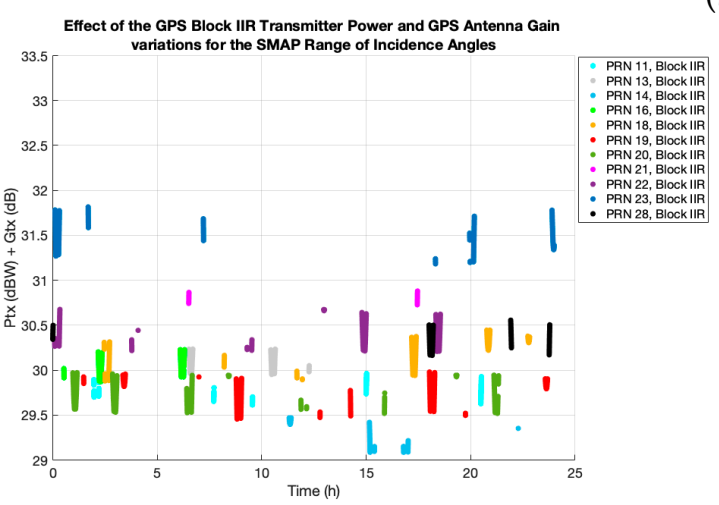

(c)

Figure 7. Combined GPS Equivalent Radiated Isotropically Power (EIRP) $\left(P_{t x} G_{t x}\right)$ for the measured range of incidence angles $\left(37.3^{\circ}\right.$ to $\left.42.7^{\circ}\right)$ and for the 32 satellites of the constellation. The main information of this plot is in the total variation range of those transmitted power. Each color corresponds to a different GPS satellite. (a) GPS block IIF satellites are represented by circles, (b) GPS block IIRM satellites are represented by squares, and (c) GPS block IIR are represented by crosses.

In addition to the reflected signal, SMAP-R also measures a direct-like signal through an antenna's back lobe. Due to its unpredictable incidence, angle is not a stable reference and is, therefore, not used for calibration. For this reason, to calibrate the GPS signal variability, we propose to collocate CYGNSS direct power and antenna gain information. Ancillary information from CYGNSS can be used for calibrating SMAP-R data by using GPS transmitted power constrained to the same day of the acquired measurements and the GPS antenna gain for the same incidence angle. By doing this, we are assuming the GPS antenna pattern is symmetric in the azimuthal direction, which leads to some remaining calibration errors. In order to evaluate the impact of this assumption, we have analyzed the combined $P_{t x}$ and $G_{t x}$ values of four GPS satellites for a period of 12 months, with results shown in 
Figure 8. A period of 12 months of observation ensures azimuthal variability in the measurements. In Figure 8, the mean variability in the vertical axis represents the antenna gain variability with respect to the incidence angle. The standard deviation of the mean variability of each GPS transmitter is due to azimuthal gain variations in its antenna pattern. The azimuthal variability has very low impact $(<0.2 \mathrm{~dB})$ on the combined $P_{t x}$ and $G_{t x}$ over a period of 12 months for the four GPS satellites analyzed. This error is much lower than the required sensitivity for most of the geophysical parameters. Therefore, the GPS transmitter information from CYGNSS metadata can be collocated with SMAP-R measurements and used as a source of information for our calibration methodology.

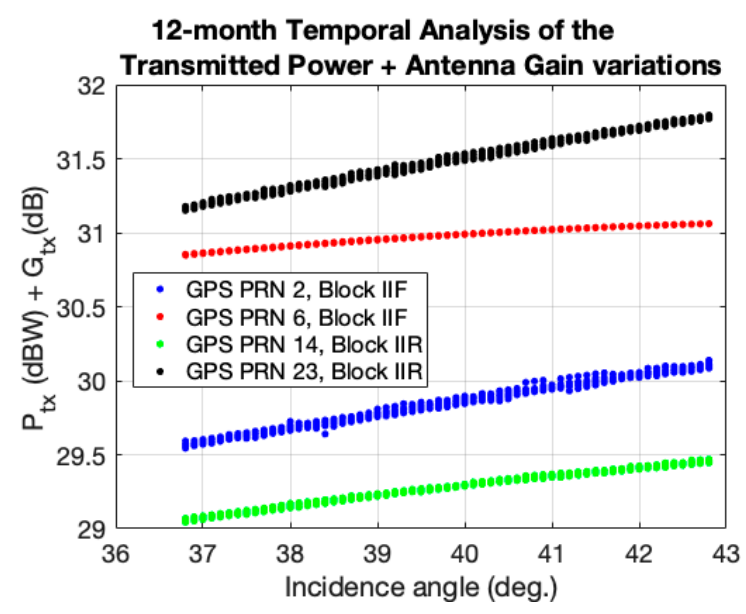

Figure 8. Temporal analysis of the variations in the combined transmitted power and gain observed for four GPS satellites over a period of 12 months. Mean variability observed in the vertical axis is due to antenna gain variations with respect to incidence angle. The standard deviation of the variability observed in the vertical axis is due to antenna gain variations with respect to azimuth angle.

\subsection{SMAP Antenna Pattern Impact}

For the SMAP antenna pattern we have assumed a linear decay of the antenna gain from the maximum at $40^{\circ}$ incidence angle to the extremes at $37.3^{\circ}$ and $42.7^{\circ}$ incidence angle from $36 \mathrm{~dB}\left(G_{\text {max }_{-} d B}\right)$ to $33 \mathrm{~dB}$ in the extremes. This follows Equation (3):

$$
G_{r x}\left(\theta_{i}\right)=G_{\max _{-} d B}-\frac{3 d B}{2.7^{\circ}} * a b s\left(40^{\circ}-\theta_{i}\right)[\mathrm{dB}]
$$

Note that $G_{r x}$ is a function of $\theta_{i}$. Corrections needed for coherently dominated scattering surfaces $(\sim 1 \mathrm{~km} \times 1 \mathrm{~km})$ are less significant than corrections needed for incoherently dominated scattering surfaces. Far from the $-3 \mathrm{~dB}$ beamwidth, the SMAP antenna gain decreases rapidly and signals coming further away from the $-3 \mathrm{~dB}$ beamwidth are expected to be greatly attenuated. As published in [42], the antenna pattern is very symmetric in azimuth within the $3 \mathrm{~dB}$ antenna beamwidth, consequently we have disregarded azimuthal variations.

\subsection{SMAP Antenna High Gain Effect}

Another important aspect of SMAP-R data is the projection of the high gain antenna beam on the scattering surface. The high gain antenna acts as a spatial filter on the total area contributing to the received power and limits the extent of the measured DDMs to those delay and Doppler bins that fall within the SMAP antenna footprint, which account only for the $-3 \mathrm{~dB}$ beamwidth with a size of $40 \mathrm{~km}$ diameter. Figure 9 shows three measurements obtained over different surfaces: Ocean (incoherent with a typical glistering zone of $\sim 250 \mathrm{~km}$, for nominal winds of $10 \mathrm{~m} / \mathrm{s}$ ), land (incoherent with a glistering zone of $\sim 5-50 \mathrm{~km}$, depends on the surface roughness), and sea ice (coherent with a typical glistering zone of $\sim 1 \mathrm{~km}$ ). 
As can be seen in Figure 9a to Figure 9c, the spread of the reflected signal varies depending on the surface type and therefore the incidence angle impact observed will be more or less critical depending on the surface type. The scattering covers a different size for each one of the DDMs shown in Figure 9. The SMAP antenna moves through this surface partially observing the GPS scattered signal, resulting in the observed DDM not being a full-DDM but a portion of it. Note that the signal does not spread significantly in the delay dimension, because of the limiting SMAP antenna footprint. Next, we describe more in further detail what is the effect of this filtering for a coherently and an incoherently dominated scattering surface.

Coherently dominated surfaces:

For surfaces as the one shown in Figure 9a-c (sea ice surface in the Kara Sea, Russia), the dominant type of scattering is coherent. Figure 10 shows measurements of a single track for three different incidence angles, $37.5^{\circ}, 40^{\circ}$ and $42.5^{\circ}$ within the $-3 \mathrm{~dB}$ beamwidth range, over some other sea ice surfaces near the Arctic, along with corresponding sketches on where the specular point is located (center of blue ellipses) versus the SMAP antenna's boresight (red dot).

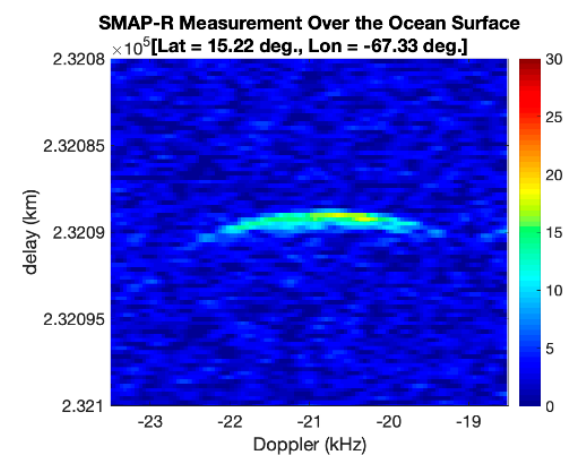

(a)

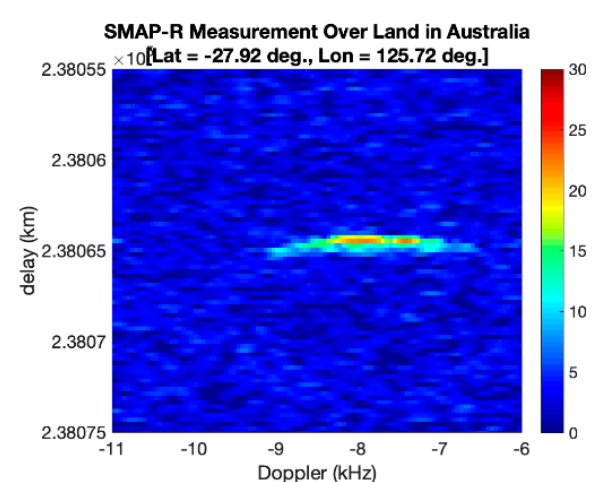

(b)

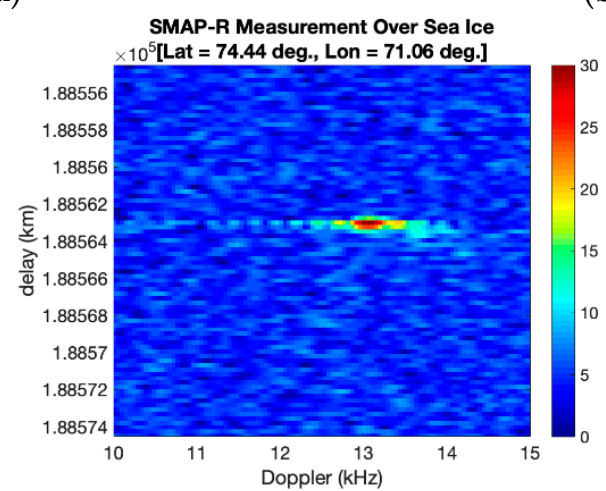

(c)

Figure 9. Delay-Doppler Maps of the scattered power measured by SMAP-R for (a) ocean surface in the edges of the Caribbean Sea between Puerto Rico and Caracas, (b) arid land surface in the middle of Australia, near the Neale Junction Nature Reserve, and (c) sea ice surface in the Kara Sea, Russia.

The effects of the SMAP high gain antenna on the measurements are small for most incidence angles and DDM bins. In other words, over the range of incidence angles considered here, the coherent scattering area typical of sea ice/lake surface $(1 \mathrm{~km} \times 1.180 \mathrm{~km})$ is within SMAP's footprint. In the incidence angle range extremes, Figure $10 \mathrm{a}$ at $37.5^{\circ}$ and Figure $10 \mathrm{c}$ at $42.5^{\circ}$, when the scattering area is near the edges of the SMAP antenna footprint-the specular point is inside the SMAP footprint, but the scattering area is only partially measured-we will observe a loss of power on the DDM. The loss of power observed in Figure 10 is $\sim 13 \mathrm{~dB}$ at both extremes and it is caused by three combined effects: 
- The SMAP antenna beam pattern at $2.5^{\circ}$ away from the boresight has an impact of $-2.8 \mathrm{~dB}$ on the total power. Note that these measurements come from the same GPS satellite.

- The GPS antenna beam pattern at the specific incidence angles causes a loss between 0.4 and $1 \mathrm{~dB}$.

- The partially measured scattering area that falls inside the SMAP antenna footprint, i.e., between 9.2 and $9.8 \mathrm{~dB}$, approximately. This partially measured scattering area causes a shape change on the DDM, since the symmetry of delay and Doppler bin cells is lost. Since the scattering area is small, the shape change in coherently dominated surfaces is only slightly noticeable.

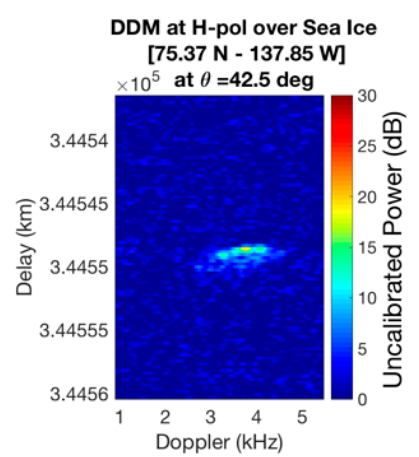

(a)

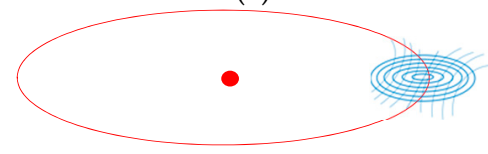

(d)

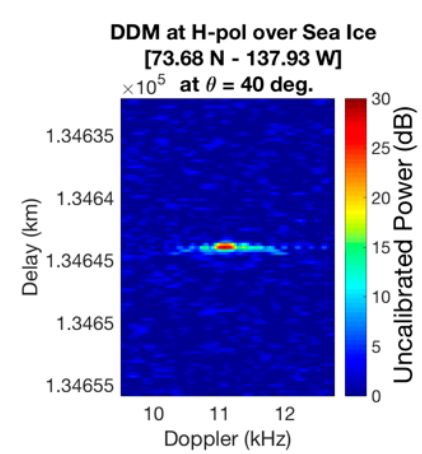

(b)

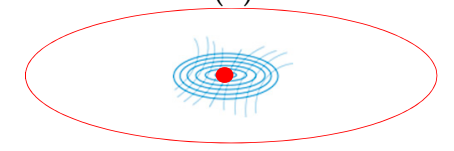

(e)

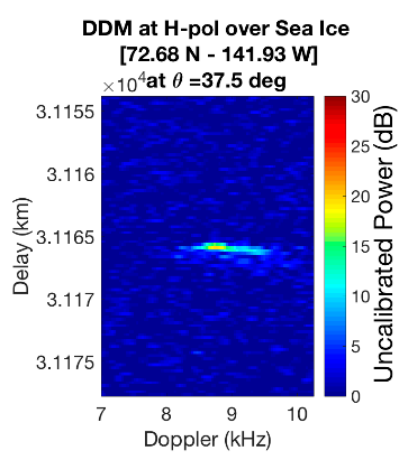

(c)

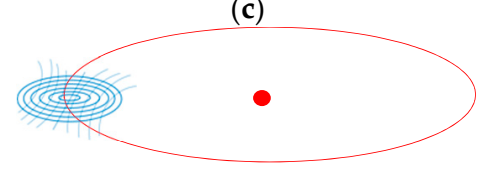

(f)

Figure 10. Top: Delay-Doppler Maps of the scattered power measured by SMAP-R for the sea ice surface for (a) $42.5^{\circ}$, (b) $40^{\circ}$, and (c) $37.5^{\circ}$ incidence angles. Bottom: iso-delay (blue ellipses) and iso-Doppler lines (blue hyperbolae) computed from SMAP-R and GPS geometries and velocities with the antenna beam pattern limited at $-3 \mathrm{~dB}$ beamwidth (red) over-imposed to those isolines for the same (d) $42.5^{\circ}$, (e) $40^{\circ}$, and (f) $37.5^{\circ}$ incidence angles. Note that they are not to scale: The scattering area represents $1 \mathrm{~km}$ major axis ellipse, while the antenna beam patter diameter is $40 \mathrm{~km}$. The antenna boresight is shown as a red dot.

Incoherently dominated surfaces:

For scenes of arid, semi-arid, or vegetated land, and for ocean areas, SMAP's narrow beam antenna plays a critical role on the scattered signal received (both strength and shape). Figure 11 shows DDM measurements for the same three incidence angles used in Figure 10, for observations over the Atlantic Ocean. Similar to Figure 10, a comparison of specular point locations (center of blue ellipses) versus the SMAP antenna's boresight (center of red circle, marked with a red dot) is provided below the DDMs.

Figure 11 illustrates how different shapes of DDMs are observed for measurements within the same track (same GPS transmitter). The shape of the DDM changes as a result of the observing geometry of the SMAP antenna in relation to the location of the reflection, i.e., the position of the specular point with respect to the antenna's boresight. Figures 11a and 11c show that for the more extreme cases, there is a loss of power on DDM peak power of $9 \mathrm{~dB}$ and a strong shape effect. Since the scattering area is only partially observed (see Figure $11 \mathrm{~d}-\mathrm{f}$ ), there is a strong asymmetry of delay and Doppler bin cells measured, exhibited by DDM shape differences with respect to incidence angle. DDM shape differences are more evident in ocean scenes, as compared to sea ice, because the scattering surface expands to sizes much larger than the SMAP antenna footprint (see Figure 11d-f). The filtering effect will become more evident as the scattered power comes from an increasingly rough surface and the surface scattering area expands from the specular point.

In order to account for the filtering effect of SMAP's antenna we will compensate for the size of the scattering area that contributed to each delay-Doppler bin in the delay-Doppler maps. Research 
conducted with SMAP-R need to consider that SMAP-R DDM shape is related mostly to the SMAP antenna beam footprint filter effect, rather than geophysical parameters alone.

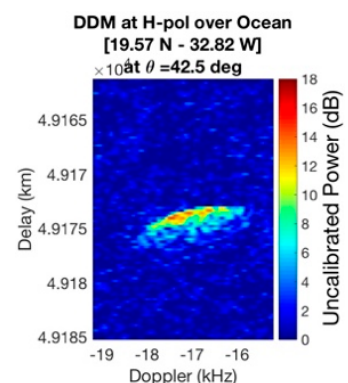

(a)

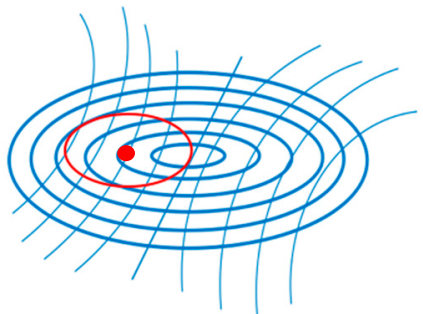

(d)

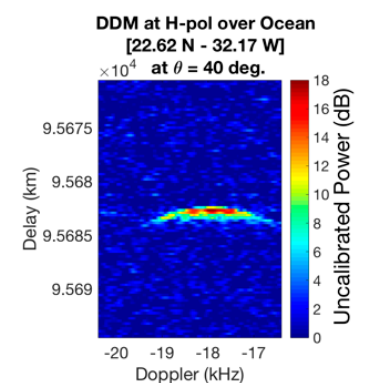

(b)

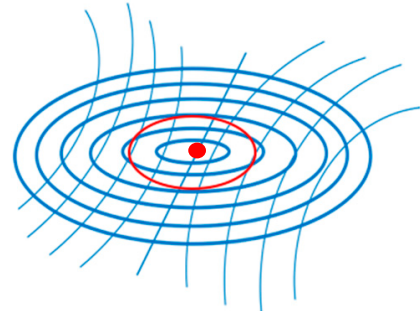

(e)

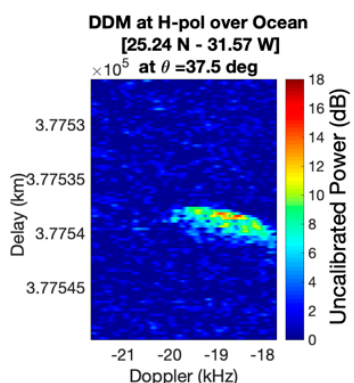

(c)

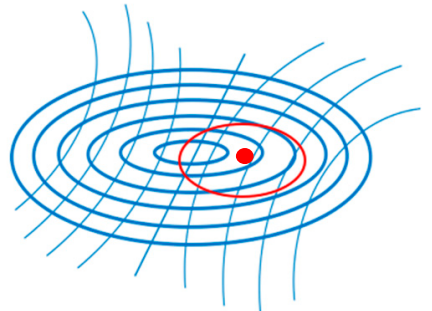

(f)

Figure 11. Top: Delay-Doppler Maps of the scattered power measured by SMAP-R for an ocean surface for (a) $42.5^{\circ}$, (b) $40^{\circ}$, and (c) $37.5^{\circ}$ incidence angles. Bottom: iso-delay (blue ellipses) and iso-Doppler lines (blue hyperbolae) computed from actual SMAP-R and GPS geometries and velocities with the antenna beam pattern (red) over-imposed to those isolines for the same (d) $42.5^{\circ}$, (e) $40^{\circ}$, and (f) $37.5^{\circ}$ incidence angles. Note that these are not to scale: The scattering area represents $200 \mathrm{~km}$ major axis ellipse, while antenna beam pattern diameter is represented by the $-3 \mathrm{~dB}$ beamwidth, i.e., $40 \mathrm{~km}$. The antenna boresight is shown as a red dot.

\subsection{SMAP-R Calibration Method}

The calibration of SMAP-R data follows Equation (1), with some specific constraints:

- $\quad T_{i}=25 \mathrm{~ms}$ for SMAP-R data, instead of the typical value of $1000 \mathrm{~ms}$ used in CYGNSS and TDS-1 missions;

- $\quad P_{t x}$ and $G_{t x}$. Extract CYGNSS GPS transmitted parameters for the same GPS satellite on the same day and for the same incidence angle as the SMAP-R measurement being processed. We also generated look-up-tables, that in case there are no coincident GPS satellite for CYGNSS and SMAP-R for that day, we can use typical values;

- $G_{r x}(\vec{\rho})$ is approximated by the gain of SMAP antenna at the specular point $G_{r x_{s p}}$ and computed from Equation (3) for the corresponding $\theta_{i}$;

- $\quad R_{r x}(\vec{\rho})$ and $R_{t x}(\vec{\rho})$ are both approximated to a constant value computed from the specular point, $R_{r x_{s p}}$ and $R_{t x_{s p}}$ respectively.

$\int \Lambda^{2}\left(\tau-\tau_{s}(\vec{\rho})\right)\left|S\left(f_{d}(\vec{\rho})-f\right)\right|^{2} d \vec{\rho}$ is the ambiguity function that includes the surface spreading in delay and Doppler for all pixels $\vec{\rho}$ within the scattering area. This can be approximated by an effective surface scattering area in the $\left(\tau, f_{d}\right)$ domain, $\bar{A}\left(\tau, f_{d}\right)$. In the case of SMAP-R, the antenna pattern projection acts as a spatial filter $F(\vec{\rho})$ in the surface domain that multiplies the surface spreading in both delay and Doppler for all pixels falling within the antenna footprint. The filtered effective surface scattering is referred as $\bar{B}\left(\tau, f_{d}\right)$. Figure 12 provides actual examples of $\bar{B}\left(\tau, f_{d}\right)$ calculations for two cases (a) $40^{\circ}$ and (b) $42.5^{\circ}$. 


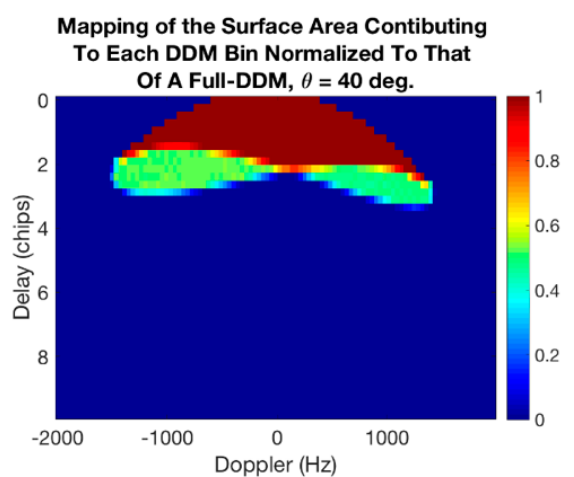

(a)

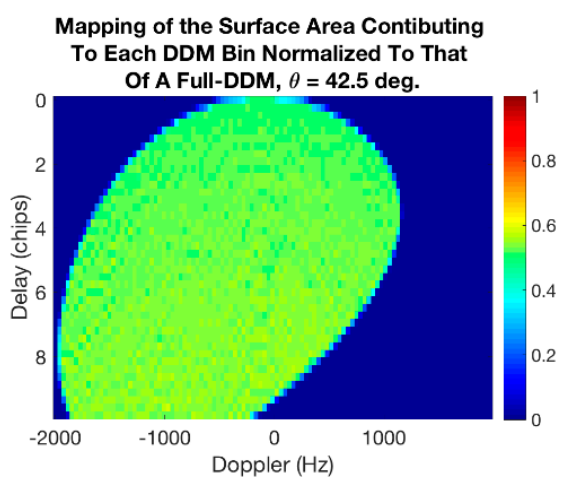

(b)

Figure 12. Spatially filtered effective surface scattering area used for (a) $40^{\circ}$ and (b) $42.5^{\circ}$ incidence angle.

In Figure 12a, delay-Doppler bins showing a value of 1 correspond to a negligible effect of the SMAP antenna, therefore the scattering area size contributing to that delay-Doppler bin is the same as if the measurement was performed with an omnidirectional antenna. Decaying delay-Doppler values shown in Figure 12, both a and b, correspond to non-negligible effect of the SMAP antenna, therefore the scattering area size contributing to that delay-Doppler bin is lower than that of a measurement acquired with an omnidirectional antenna. To compute $\bar{B}\left(\tau, f_{d}\right)$, we followed these steps:

1. Convert the transmitter and receiver position and velocity coordinates from Earth Centered Earth Fixed (ECEF) to the specular frame;

2. Define a fine grid;

3. Calculate the delay and Doppler values over that grid;

4. Compute the size of the surface patches at each delay-Doppler grid set by SMAP-R DDM bins;

a. Compute those surface patch sizes using the SMAP antenna footprint (40 km diameter) located at variable distances from the specular point (incidence angle of $40^{\circ}$ is centered with the specular point, incidence angles smaller or larger are not centered $)=B\left(\tau, f_{d}\right)$;

b. Compute the surface patch sizes with no filter (i.e., filtering effect compensation) $=A\left(\tau, f_{d}\right)$;

5. Compute the compensating factor for the filtered scattering surfaces as $\bar{B}\left(\tau, f_{d}\right)=\frac{B\left(\tau, f_{d}\right)}{A\left(\tau, f_{d}\right)}$.

In the examples shown in Figure 12, in the case of $40^{\circ}$ incidence angle, most of the delay-Doppler bins near the specular point are not affected by the SMAP antenna footprint and, as the delay increases, the filtering effect starts to be noticeable. In the case of $42.5^{\circ}$, the effect is noticeable for all DDM bins, including the bin corresponding to the DDM peak. Therefore, in order to make consecutive measurements that are comparable with information from all incidence angles there is a need to compensate for the loss of power caused by a filtered scattering surface. Also, note that the mapping of the surface area contributing to each delay-Doppler map bin has the same shape at that observed in the scattering of the measurements in Figures $11 \mathrm{~b}$ and $11 \mathrm{a}$, for $40^{\circ}$ and $42.5^{\circ}$, respectively. $\bar{B}\left(\tau, f_{d}\right)$ has been precomputed for multiple geometries, from $37.3^{\circ}$ to $42.7^{\circ}$ incidence angles in steps of $0.1^{\circ}$.

The resulting calibration equation is shown in Equation (4) and Equation (5), i.e., the radar cross section $\sigma_{0}$, which is the same equation used in CYGNSS calibrations but slightly modified to the SMAP-R characteristics, for the incoherent and coherent assumptions.

$$
\begin{gathered}
\sigma_{0 \text { incoh }}=\frac{(4 \pi)^{3} P_{\text {incoh }}\left(\tau, f_{d}\right) R_{r x_{s p}}^{2} R_{t x_{s p}}^{2}}{T_{i}^{2} P_{t x} G_{t x} \lambda^{2} G_{r x_{s p}} \bar{B}\left(\tau, f_{d}\right)} \\
\sigma_{0 \text { coh }}=\frac{(4 \pi)^{3} P_{\text {incoh }}\left(\tau, f_{d}\right)\left(R_{r x_{s p}}+R_{t x_{s p}}\right)^{2}}{T_{i}^{2} P_{t x} G_{t x} \lambda^{2} G_{r x_{s p}} \bar{B}\left(\tau, f_{d}\right)},
\end{gathered}
$$




\section{Calibrated Data Quality Analysis}

To validate this calibration approach, we have used a vicarious surface calibration target. In order to reduce the uncertainty within a scene, i.e., the actual variability due to geophysical properties of the surfaces, we required surfaces with large areas of constant observational conditions for a prolonged time. Observations dominated by coherent scattering, with smaller scattering areas $(\sim 1 \mathrm{~km} \times 1 \mathrm{~km})$, are useful because they have less spatial variability. The expected scene variability varies with the type of scattering surface. For example:

- Land surfaces have a large scene composition variability with convoluted geophysical parameters characterization that impact the power reflected from those areas. For example, two specular points side by side will not have the same power;

- Ocean surfaces are generally rough. The roughness varies with the wind speed intensity and the wind direction (which can change quickly). Measurements from the ocean are therefore not constant over long periods of time;

- Sea ice surfaces are highly coherent and are usually characterized by low variability characteristics over large areas. Particularly in the Arctic, large areas of old sea ice are formed and remain stable during winter months;

- A global surface coherency mapping was developed by authors in [35,36] using TDS-1. Results showed that between June and August, the most coherent results come from the sea ice surrounding the Antarctica, the Sahara Dessert, and Australia, while between November and March, the most coherent results come from Arctic sea ice, the Sahara Dessert, and Australia;

Expected calibration effect:

The calibration surface target selected are extensive areas of old sea ice in the Arctic, with minimal presence of first year ice towards the Alaskan coast, during the month of February in 2018. At this time, the sea ice was well established with constant $100 \%$ concentration. A research study [18] indicated that old sea ice is the least reflective type of sea ice when compared to thinner ice such as pancake ice or new ice. Ice type has an impact on the reflectivity and therefore the DDM peak SNR, as it was shown in [18]. That study also showed that young ice has higher reflectivity since it tends to be smoother. Old ice is rougher and shows lower reflectivity as compared to new ice. However, old ice is usually more stable over long periods of time and covers a larger portion of the sea ice. The expected DDM power will be higher than ocean areas $(>15 \mathrm{~dB}$ - uncalibrated levels) and lower than the more reflective young sea ice ( $<30 \mathrm{~dB}$ - uncalibrated levels). Power levels should therefore range between $10-12 \mathrm{~dB}$ for the more reflective ice (i.e., $>18 \mathrm{~dB}$ - uncalibrated levels). The calibration performed in this study is expected to preserve the variability of the scene due to differences in the sea ice, while correcting for the GPS EIRP dependence as well as the incidence angle dependence derived from the spatial filtering effect of the high gain SMAP antenna.

Figures 13 and 14 illustrate the two described characteristics of the selected calibration surface target: 100\% sea ice concentration of old ice and first year ice types. These characteristics ensure coherent reflections, i.e., a limited scattering area size to something close to $\sim 1 \mathrm{~km} \times 1 \mathrm{~km}$, and no presence of open water. Figure 13 shows the Arctic sea ice coverage during the two extremes of a stable time period.

Figure 13 shows that the sea ice coverage remains nearly constant from December to March-2017/2018 winter season, and the ice concentration is 100\% for the entire period for most of the area. Figure 14 shows the dominant ice type of the area selected as our calibration target (white dotted box).

Figure 14 clearly shows that the Alaskan coast is covered by first year ice (orange), while the rest of the area is covered by old ice (brown). Figure 14 also identifies the dominant ice type, which indicates that over $50 \%$ of the area was covered by the indicated particular ice. There is therefore a possibility that the indicated sea ice type might actually be a mixture of different sea ice types and the GPS signals measures this variability, [18]. 


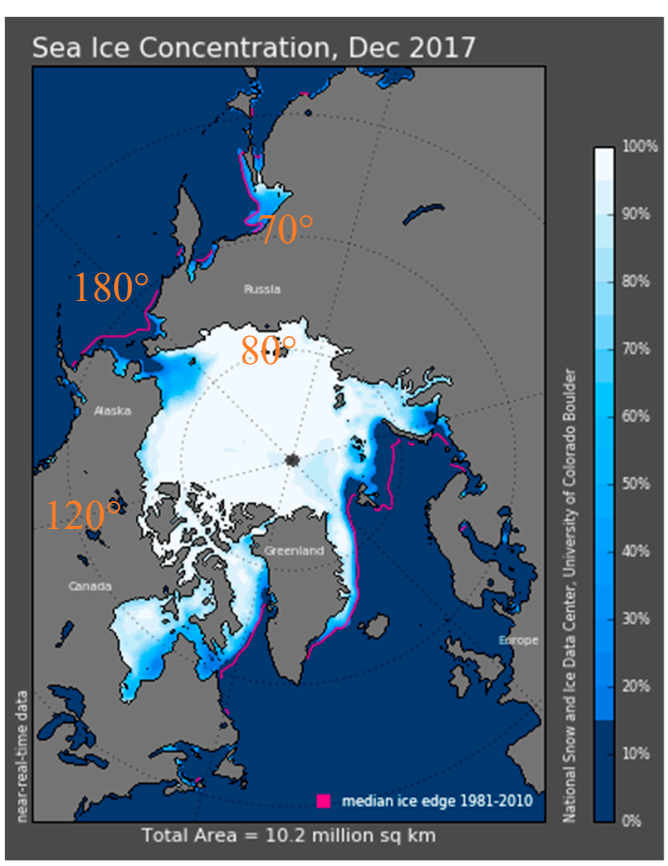

(a)

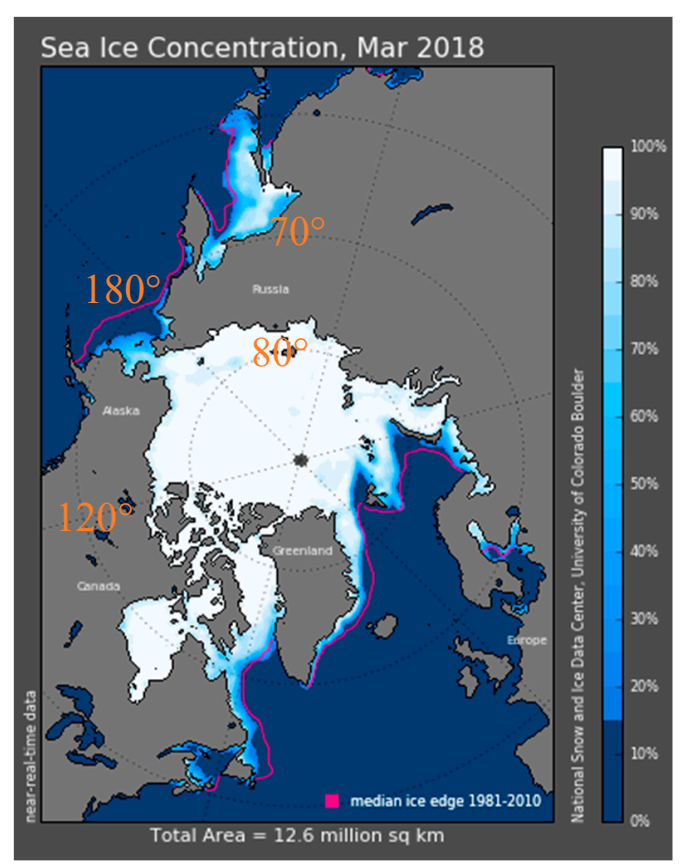

(b)

Figure 13. Arctic sea ice concentration reported by the National Snow and Ice Data Center [43], University of Colorado Boulder on (a) December 2017 and (b) March 2018. Ice concentration remains constant since December to March. Images courtesy of the National Snow and Ice Data Center (NSIDC), University of Colorado, Boulder. The images are derived from Sea Ice Index NSIDC data product, which relies on NASA-developed methods using passive microwave data from the Defense Meteorological Satellite Program (DMSP) F-18 Special Sensor Microwave Imager/Sounder (SSMIS). The Sea Ice Index [44] was developed by the NSIDC with financial support from NOAA NESDIS and in cooperation with NOAA NGDC. Color scale from $0 \%$ (dark blue) to $100 \%$ (white) on $10 \%$ increments.

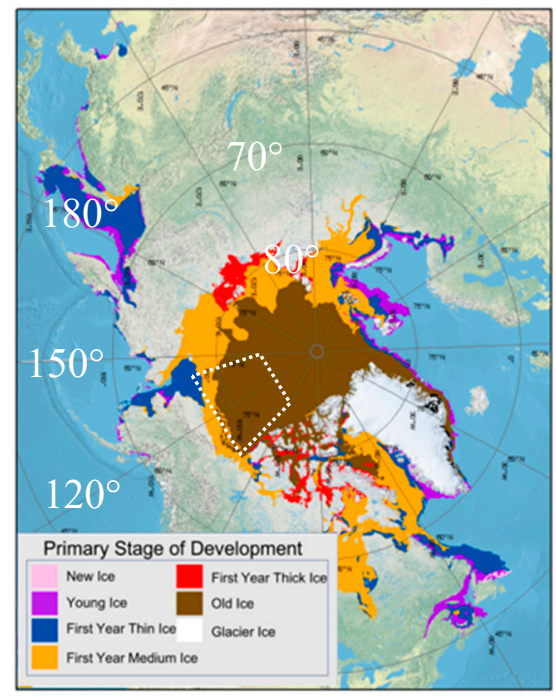

Figure 14. Arctic sea ice primary stage of development obtained from the NOAA National Ice Center website (https://www.natice.noaa.gov) for February 2018. The brown color corresponds to old ice, and red, orange and blue color correspond to three types of first year ice. Pink color corresponds to new ice and purple color to young ice. The white dotted box defines the calibration target area selected for this study. 


\section{Calibration}

Equation (5) has therefore been applied to a large dataset of samples gathered in February 2018 within the selected area, accounting for the coherency of the reflected signals. The results of the calibration methodology are next evaluated.

A. Figure 15 shows the differences observed on the received powers due to differences on the GPS EIRP. Data corresponds to February 2018 over the calibration selected area in the Arctic Chukchi-Beaufort Sea area.

Figure 15 shows that different tracks measured from different GPS transmitters need corrections that can differ from $-2 \mathrm{~dB}$ to $2 \mathrm{~dB}$ from a reference GPS EIRP of $31.5 \mathrm{~dB}$ (mean of all GPS EIRP in Figure 6). We compared the dependence of the uncalibrated data and the calibrated data to the differences in the GPS EIRP. In order to verify the effect of calibrating the GPS transmitted EIRP (Ptx + Gtx) (dB), we need to select a pixel and observe its power return though time. We assumed that the surface conditions remained nearly constant and consequently a given pixel should have relatively constant signal power return (low variability) during consecutive measurements (in consecutive days). Because of the SMAP-R surface sampling configuration, observing the same pixels is unlikely. Therefore, to ensure multiple observations in time, instead of selecting a single pixel on the surface, we selected a small area (south-west area within our calibration target area). The caveat with this approach is that the variability within the scene is included in the signal, however, it ensures that enough measurements are available to analyze the time series variability of the GPS transmitter information. Figure 16 shows the effect of calibrating the differences in GPS transmitted EIRP (dB).

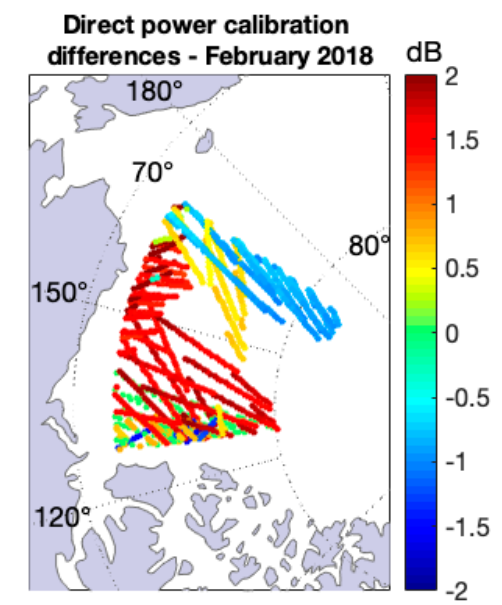

Figure 15. Impact of the direct signal power and gain differences between GPS satellites for February 2018 data. Up to $4 \mathrm{~dB}$ total difference correction is observed from minimum to maximum values, which applies to both H-pol and V-pol.

We selected a reduced area within our calibration target area (south-west corner) that has been observed with a wide range of GPS satellite EIRP's over a number of days. The uncalibrated data (Figure 16a) shows a larger slope than the calibrated data (Figure 16b). A slope of 1.08 over a range of EIRP's $(\mathrm{dB})$ of about $4 \mathrm{~dB}$, causes differences of $4.32 \mathrm{~dB}$ in the mean. After calibration, those differences have been reduced to $0.8 \mathrm{~dB}$ in the mean (slope of $-0.2 \mathrm{~dB}$ ) achieving an $81.5 \%$ reduction on the mean bias, with a residual error of $0.8 \mathrm{~dB}$. The residual error possibly originates from the variability of the scene itself, CYGNSS information inaccuracies, and the actual azimuthal differences in the GPS antenna gain patterns not accounted for in this study. Overall the calibration produces a flatter response, with a low dependence on the GPS transmitter information reduced from a mean value of $4.32 \mathrm{~dB}$ to a mean value of $0.8 \mathrm{~dB}$. 


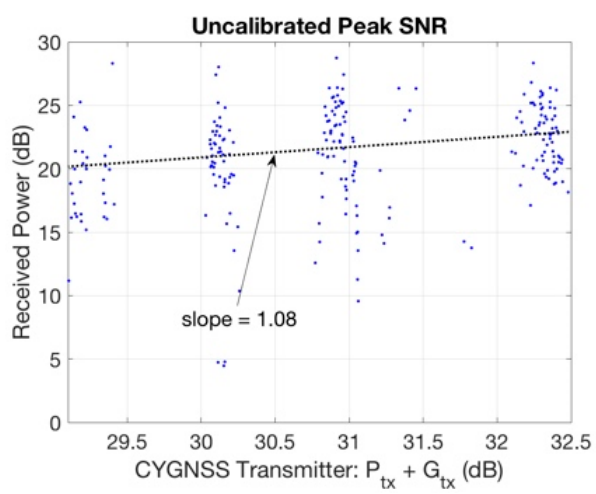

(a)

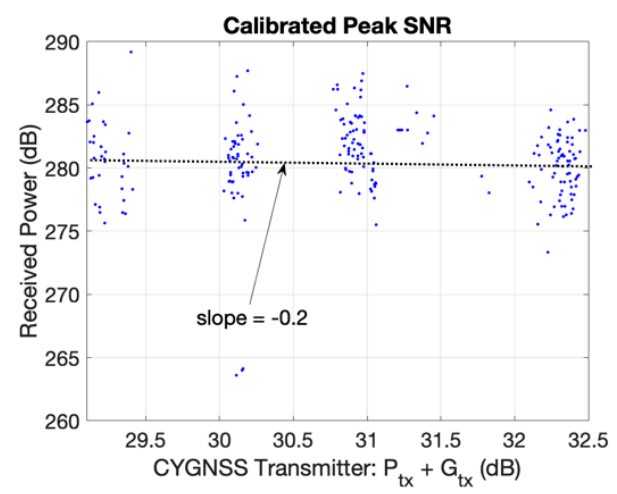

(b)

Figure 16. February 2018 SMAP-R samples as a function of the GPS transmitter information $\left(P_{t x}+G_{t x}\right)$ (a) uncalibrated data over a small area of the calibration target area (south-west corner) and (b) calibrated data over the same small area of the calibration target area. Note that calibrated values correspond to radar cross section values after applying calibration Equation (5), under coherent assumption.

B. Figure 17 shows the result of applying a correction to the filtering effect of the SMAP high gain antenna to same data from February 2018.

Figure 17 shows a power variability from 0 to $-10 \mathrm{~dB}$ in the peak SNR. Most of the time the error is low, but there are some samples showing a sudden increase in variability. Those samples of higher variability correspond to consecutive measurements with incidence angles further away from the SMAP antenna pointing at $40^{\circ}$. This reduction in SNR is therefore due to the SMAP high gain antenna filtering effect on the surface area corresponding to the DDM peak delay and Doppler bin. Similar information is plotted in Figure 18, with corrections applied to the peak SNR as a function of the incidence angle for February 2018.

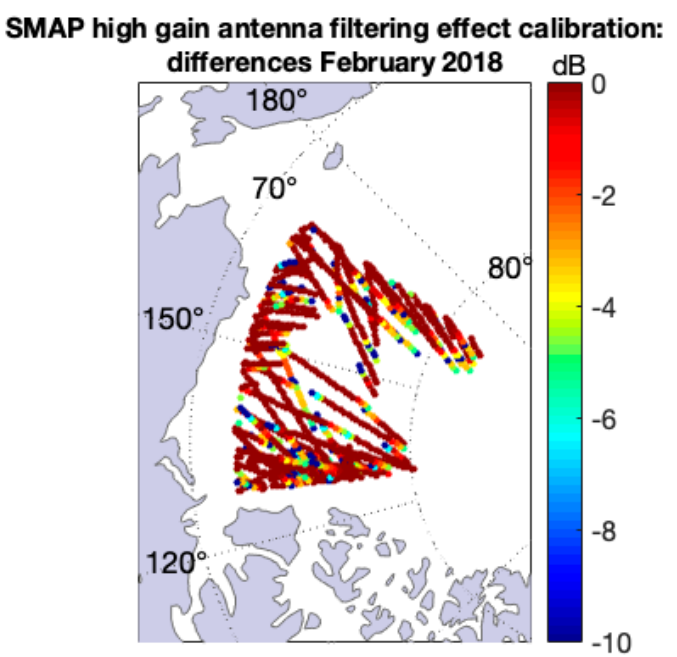

Figure 17. Impact of the SMAP antenna high gain filtering effect calibration for February 2018 data. $\mathrm{Up}$ to $10 \mathrm{~dB}$ total difference correction is observed. The larger errors correspond to reflections farther away from the SMAP antenna boresight. This correction applies to both H-pol and V-pol.

Figure 18 shows that the larger corrections correspond to reflections farther away from the SMAP antenna boresight. When considering only the delay-Doppler bin of the DDM peak, no correction is needed for incidence angles close to the antenna boresight $\left(40^{\circ}\right)$. For reflections with incidence angles below $38.4^{\circ}$ and over $41.6^{\circ}$ incidence angle, relevant corrections are needed. The correction shown in Figure 18 only applies to peak SNR. To calibrate the whole DDM, the corresponding scattering area normalization needs to be applied to each DDM delay and Doppler bin individually, as shown in 
Figure 12. The effect of this calibration is shown in Figure 19, where the dependence of the uncalibrated data and the calibrated data to the incidence angle is analyzed. The correction performed to account for the filtering effect of the SMAP high gain antenna is expected to reduce the dataset dependence on incidence angle. Therefore, in order to verify the effect of calibrating the SMAP high gain antenna effect, the whole dataset is plotted as a function of the incidence angle.

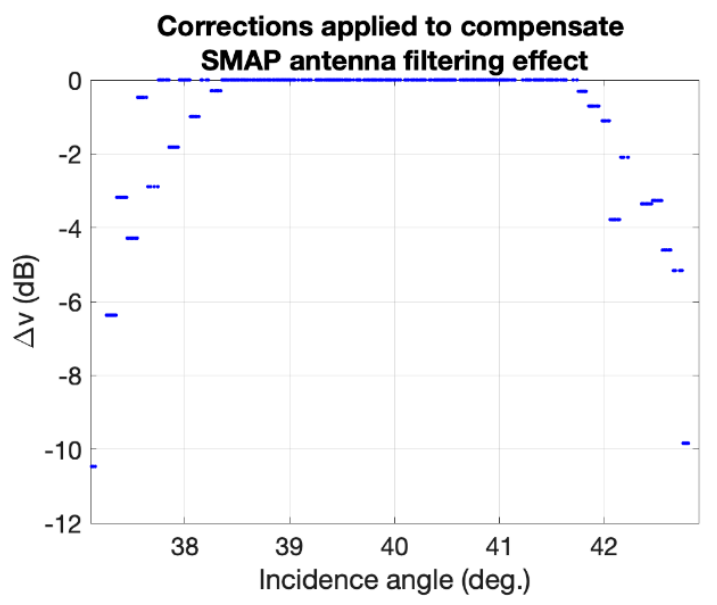

Figure 18. Corrections applied to peak SNR as a function of the incidence angle for February 2018. Corrected peak SNR $=$ peak SNR $-\Delta v$.

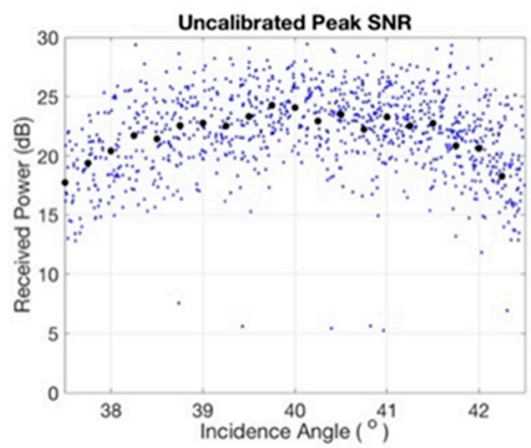

(a)

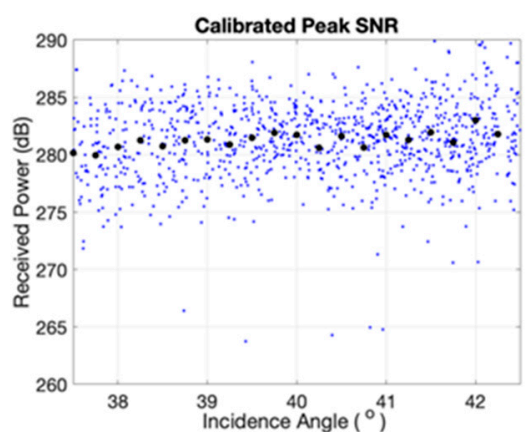

(b)

Figure 19. February 2018 SMAP-R samples as a function of the high gain antenna incidence angle: (a) uncalibrated and (b) calibrated. Note that calibrated values correspond to radar cross section values after applying calibration Equation (5), under coherent assumption.

Figure 19a shows the uncalibrated area following a curvature similar to what is expected from Figure 18. The curvature towards the incidence angles away from $40^{\circ}$ is calibrated in Figure $19 \mathrm{~b}$, showing a non-incidence angle dependency for the whole dataset. The uncalibrated dataset shows a total variability of $7 \mathrm{~dB}$, including mean angular dependency and data dispersion. It shows a curved signature dependency with the incidence angle. However, the calibrated data shows no angular dependency with $2.5 \mathrm{~dB}$ dispersion. Although the curvature angular dependency is removed, the calibrated data shows some degree of dispersion $(2.5 \mathrm{~dB})$ that is part of the intrinsic fluctuations of the scene itself together with the GPS transmitter inaccuracy corrections $(\sim 0.8 \mathrm{~dB})$.

\section{Calibration Results:}

The calibration was applied to SMAP-R samples acquired during February 2018. Figure 20 shows the variability of the uncalibrated and calibrated measurements at both V-polarization (top) and the H-polarization (bottom) over old ice areas February 2018. 


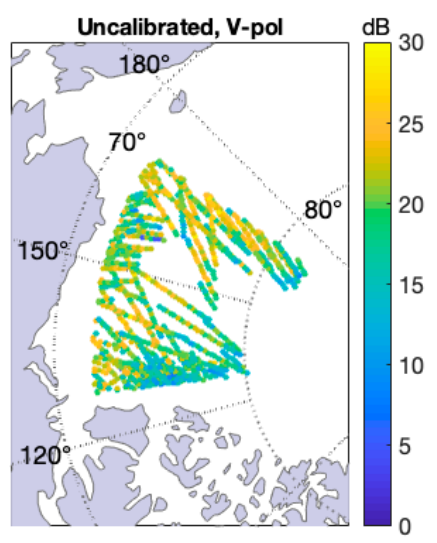

(a)

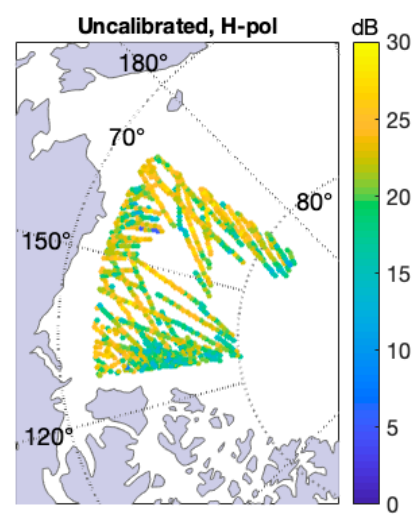

(c)

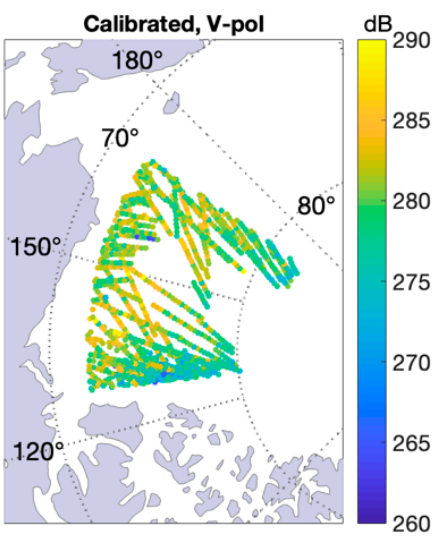

(b)

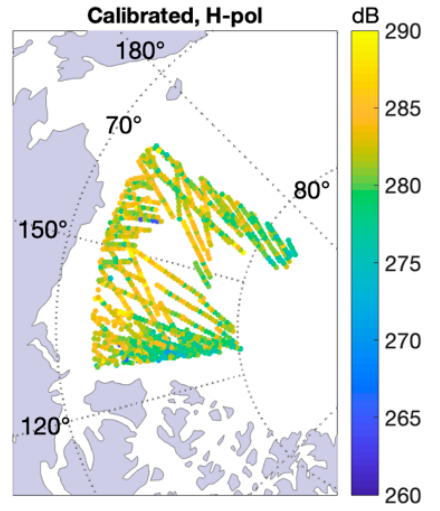

(d)

Figure 20. SMAP-R measurements for February 2018, (a) uncalibrated V-pol and (b) calibrated V-pol, and (c) uncalibrated H-pol and (d) calibrated H-pol. Calibration includes both the correction of the GPS transmitter variability (collocating direct power information from CYGNSS) and the correction of the effect of the SMAP high gain antenna. Note that calibrated values correspond to radar cross section values after applying calibration Equation (5) under a coherent assumption.

The dominant ice type within the study area is old ice (see Figure 13), although first year ice is present in the south-west corner of the area selected and the Alaskan coast. Dominant ice type in Figure 13 means that over $50 \%$ of the analyzed pixels were covered by the assigned class. Therefore, the presence of open water or other classes of sea ice is possible. Since our measurements are based on forward scattering, the presence of first year ice will cause an increase in the coherency of the reflection in our observations and open water will contribute to rougher observations. Figure 12 shows that the sea ice concentration was $100 \%$ for February 2018, which give us confidence that no or minimal open water was present within our calibration target area. This is supported by peak SNR indicating that there were only five samples with $<10 \mathrm{~dB}$ SNR, which we assume were open water. Figure 20 shows that the calibration has not reduced or removed the variability within the scene (due to nondominant presence of first year ice within cells of dominant old ice). The calibration has only corrected for the differences caused by the GPS transmitter EIRP variability and the effect of the SMAP high gain antenna on the peak SNR. Overall, the calibrated data is more spatially consistent as shown in Figure 20. Figure 21 illustrates a formal spatial variability analysis.

The spatial variability has been computed as the standard deviation of the measurements in boxes of $1^{\circ}$ latitude $\times 1^{\circ}$ longitude. Figure 21 shows that the calibrated data, both at H-pol and V-pol, have a reduced spatial variability relative to the uncalibrated data. The highest spatial variability observed in the uncalibrated data is close to $11 \mathrm{~dB}$, which is reduced in the calibrated version around $\sim 2-3 \mathrm{~dB}$. The high spatial variability of the uncalibrated samples was caused by the two effects corrected in the 
calibration process (differences between transmitted EIRP and filtering effect of the SMAP high-gain antenna) and, therefore, did not correspond to any given geophysical feature within the scene.

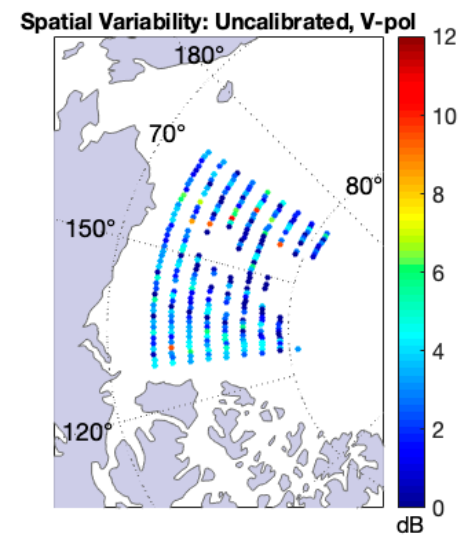

(a)

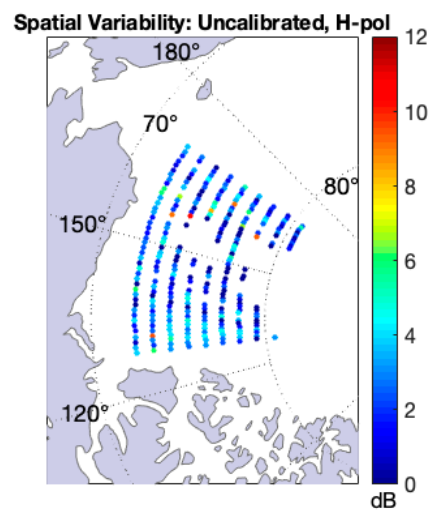

(c)

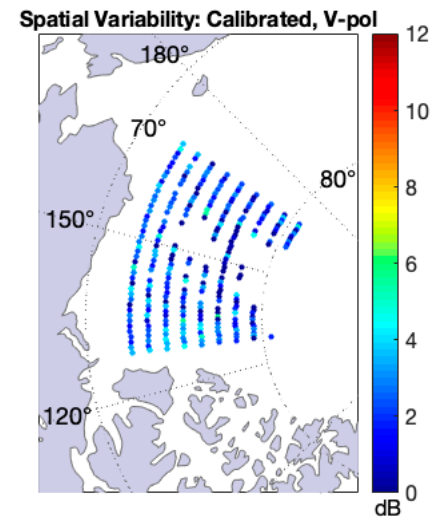

(b)

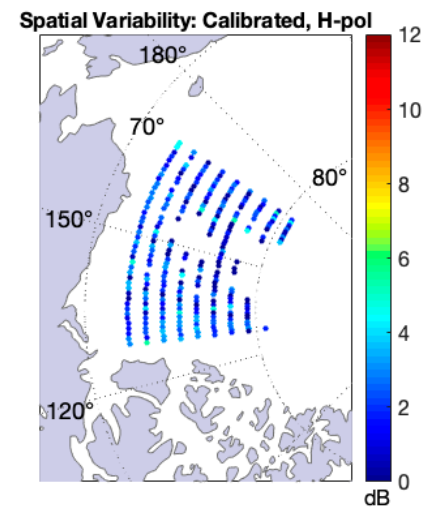

(d)

Figure 21. Standard deviation representing the spatial variability observed for February 2018 data: (a) uncalibrated V-pol, (b) calibrated V-pol, (c) uncalibrated H-pol, and (d) calibrated H-pol. The spatial variability is analyzed in $1^{\circ} \times 1^{\circ}$ boxes (lat/lon). Note that calibrated values correspond to radar cross section values after applying calibration Equation (5), under coherent assumption.

\section{Discussion}

The calibration of the SMAP-R dataset is a key step in the production and delivery of this data to the scientific community. Results show that when using the peak SNR of the DDMs alone, the differences between using the calibrated and the uncalibrated SMAP-R data can be up to $12 \mathrm{~dB}$ for samples with incidence angles below $38.4^{\circ}$ and over $41.6^{\circ}$. This effect is even more critical in the delay and Doppler bins further away from the peak one. We demonstrated that those differences have an impact on the sensitivity to different geophysical parameters, which can mislead the results obtained in scientific research.

The characteristics of SMAP-R, such as its high antenna gain, polarimetric measurements $(\mathrm{H}$ and $\mathrm{V}$ polarizations), and polar coverage, provide the capability to study cryosphere, land, and ocean processes such as sea ice properties, land surface, freeze/thaw state, freeze/thaw seasonal transition, and vegetation water content. The low integration times characteristic of SMAP-R measurements could also benefit studies related to oil slick detection, which are characterized by highly coherent signatures over the ocean surface, but the low temporal coverage reduces the possibility to observe such events. SMAP-R could also enhance studies of wetlands and inundation. The availability of raw data also opens the possibility for analysis of the phase of the measurements. For example, highly coherent surfaces such as the sea ice will allow phase altimetry studies to determine the thickness of the ice [17]. The greatest challenge is the low sampling and low coverage of SMAP-R, which limits 
some of the applications requiring high repeatability and daily coverage over small areas (such as flooding or oil slicks). Other applications that do not require high temporal repeat over small areas can be addressed through observational strategies by averaging data over longer periods of time (15 days to 1 month) to obtain two state extremes for a given geophysical parameter and analyzing the seasonal transitions between states.

SMAP-R is a great asset for understanding the capabilities that global L-band polarimetric measurements can bring to the scientific community. SMAP-R is based on forward scattering, which could complement future active radar satellite missions such as the NASA-ISRO SAR Mission (NISAR, [45]). NISAR is a joint SAR satellite mission between NASA and the Indian Space Research Organization (ISRO) that is optimized for studying hazards and global environmental change. It will measure backscatter at L- and S-bands at dual polarization, with polar coverage and will provide 12 days exact repeat cycles with a spatial resolution of 3-10 m (mode dependent). Daily updates from SMAP-R can be used to fill in the gaps between NISAR repeat cycles. SMAP-R is also a great asset towards the development of future GNSS-R missions, by informing on the potential of polarimetric measurements, global coverage, phase information, higher SNRs, and reduced integration times.

\section{Conclusions}

This manuscript describes the specific characteristics of SMAP-R dataset and how it compares to CYGNSS and TDS-1 missions' measurements. The calibration of the SMAP-R dataset is shown more challenging than the calibration required by other GNSS-R missions specifically designed for the technique. In particular, this manuscript addresses both the lack of direct power information and the filtering effect of the high gain antenna. While consideration for the SMAP antenna pattern is important for SMAP-R, the antenna filtering effect minimally impacts other missions. For example, CYGNSS may observe a similar effect at high incidence angles, as the scattering area extends outside of the CYGNSS antenna beam pattern. In general, only a few delay-Doppler bins around the specular point are considered as input for estimates of geophysical phenomena; near the specular point, antenna filtering effects are of less concern.

This manuscript describes the calibration methodology applied to SMAP-R measurements and provides an analysis of the quality of the calibration for a small region characterized by constant old ice. We selected this area to ensure coherent returns (reduced scattering areas) with lower scene variability, which allowed us to better explore the particular corrections performed in our study. Other targets could had been considered, specifically other surfaces characterized by large extensions of coherent scattering. The Sahara Desert, big lakes around the globe, or areas of Australia are some examples of those areas. A standardized methodology can therefore employ the calibration presented in this manuscript performing quality analysis over those surfaces on a regular basis.

Our results show that the calibration methodology applied to correct the dependence on GPS transmitter information achieves an $81.5 \%$ reduction on the mean bias. The methods presented here reduce the variability observed in the uncalibrated dataset $(4.2 \mathrm{~dB})$ to a variability of $0.8 \mathrm{~dB}$ in the calibrated dataset. Also, the calibration methodology presented corrects the dependence of the SMAP-R dataset with incidence angle, reducing the mean dispersion from $7 \mathrm{~dB}$ to $2.5 \mathrm{~dB}$. This correction is obtained by compensating the filtering effect of the SMAP high gain antenna. Finally, the calibrated data shows an overall reduction in the mean spatial variability of $2-3 \mathrm{~dB}$ and a reduction in the areas of high spatial variability that were observed with the uncalibrated data.

Future work will include the calibration of the entire DDMs. The calibration will be done applying corrections similar to the one shown in Figure 11 to each bin of the DDM. Or equivalently, applying similar corrections to the ones shown in Figure 17 at each delay-Doppler bin of the DDM. In addition, to eliminate sources of error in the calibration, future work should employ CYGNSS version 3.0 data to obtain more accurate EIRP values as well as perform a subday collocation between SMAP measurements and CYGNSS-reported transmitted powers to avoid averaging out relevant variations 
due to GPS flex mode applied to the different blocks. Future work will also include the development of new science products from the calibrated dataset.

Author Contributions: Conceptualization, N.R.-A., S.M., E.P., M.M. and X.B.-L.; methodology, N.R.-A.; software, N.R.-A.; validation, N.R.-A.; formal analysis, N.R.-A.; investigation, N.R.-A.; writing-original draft preparation, N.R.-A.; writing-review and editing, N.R.-A., S.M., E.P., M.M. and X.B.-L.; visualization, N.R.-A. and M.M.; funding acquisition, E.P. and S.M.

Funding: This research was carried out at the Jet Propulsion Laboratory, California Institute of Technology, under a contract with the National Aeronautics and Space Administration. The research was supported by a JPL fund on R\&A Hydrology \& Weather from the Soil Moisture Active Passive (SMAP) project and by a JPL Research and Technology Development (R\&TD) Grant (PI-E. Podest). (C) 2019. California Institute of Technology. Government sponsorship acknowledged.

Acknowledgments: The SMAP-R data analysis has been possible thank to the earlier efforts conducted at JPL by Stephen Lowe and Stephan Esterhuizen in which a processor was developed to take the I/Q samples of SMAP radar receiver and process them to the stage of uncalibrated DDMs.

Conflicts of Interest: The authors declare no conflict of interest.

\section{References}

1. Piepmeier, J.R.; Focardi, P.; Horgan, K.A.; Knuble, J.; Ehsan, N.; Lucey, J.; Brambora, C.; Brown, P.R.; Hoffman, P.J.; French, R.T.; et al. SMAP L-Band Microwave Radiometer: Instrument Design and First Year on Orbit. IEEE Trans. Geosci. Remote Sens. 2017, 55, 1954-1966. [CrossRef]

2. Unwin, M.; Jales, P.; Tye, J.; Gommenginger, C.; Foti, G.; Rosello, J. Spaceborne GNSS-Reflectometry TechDemoSat-1: Early Mission Operations and Exploitation. IEEE J. Sel. Top. Appl. Earth Obs. Remote Sens. 2016, 9, 4525-4539. [CrossRef]

3. Ruf, C.S.; Gleason, S.; Jelenak, Z.; Katzberg, S.; Ridley, A.; Rose, R.; Scherrer, J.; Zavorotny, V. The CYGNSS nanosatellite constellation hurricane mission. In Proceedings of the 2012 IEEE International Geoscience and Remote Sensing Symposium, Munich, Germany, 22-27 July 2012; pp. 214-216.

4. Ruf, C.; Unwin, M.; Dickinson, J.; Rose, R.; Rose, D.; Vincent, M.; Lyons, A. CYGNSS: Enabling the Future of Hurricane Prediction [Remote Sensing Satellites]. IEEE Geosci. Remote Sens. Mag. 2013, 1, 52-67. [CrossRef]

5. Ruf, C.S.; Atlas, R.; Chang, P.S.; Clarizia, M.P.; Garrison, J.L.; Gleason, S.; Katzberg, S.J.; Jelenak, Z.; Johnson, J.T.; Majumdar, S.J.; et al. New Ocean Winds Satellite Mission to Probe Hurricanes and Tropical Convection. Bull. Am. Meteorol. Soc. 2016, 97, 385-395. [CrossRef]

6. Clarizia, M.P.; Ruf, C.S.; Jales, P.; Gommenginger, C. Spaceborne GNSS-R Minimum Variance Wind Speed Estimator. IEEE Trans. Geosci. Remote Sens. 2014, 52, 6829-6843. [CrossRef]

7. Foti, G.; Gommenginger, C.; Jales, P.; Unwin, M.; Shaw, A.; Robertson, C.; Roselló, J. Spaceborne GNSS reflectometry for ocean winds: First Results from the UK TechDemoSat-1 mission. Geophys. Res. Lett. 2015, 42, 5435-5441. [CrossRef]

8. Clarizia, M.P.; Ruf, C.; Cipollini, P.; Zuffada, C. First spaceborne observation of sea surface height using GPS-Reflectometry. Geophys. Res. Lett. 2016, 43, 767-774. [CrossRef]

9. Chew, C.; Shah, R.; Zuffada, C.; Hajj, G.; Masters, D.; Mannucci, A.J. Demonstrating soil moisture remote sensing with observations from the UK TechDemoSat-1 satellite mission. Geophys. Res. Lett. 2016, 43, 3317-3324. [CrossRef]

10. Camps, A.; Park, H.; Pablos, M.; Foti, G.; Gommenginger, C.P.; Liu, P.W.; Judge, J. Sensitivity of GNSS-R Spaceborne Observations to Soil Moisture and Vegetation. IEEE J. Sel. Top. Appl. Earth Obs. Remote Sens. 2016, 9, 4730-4742. [CrossRef]

11. Nghiem, S.V.; Zuffada, C.; Shah, R.; Chew, C.; Lowe, S.T.; Mannucci, A.J.; Cardellach, E.; Brakenridge, G.R.; Geller, G.; Rosenqvist, A. Wetland monitoring with Global Navigation Satellite System reflectometry. Earth Space Sci. 2017, 4, 16-39. [CrossRef]

12. Zuffada, C.; Chew, C.; Nghiem, S.V. Global navigation satellite system reflectometry (GNSS-R) algorithms for wetland observations. In Proceedings of the 2017 IEEE International Geoscience and Remote Sensing Symposium (IGARSS), FortWorth, TX, USA, 23-28 July 2017; pp. 1126-1129.

13. Yan, Q.; Huang, W. Spaceborne GNSS-R Sea Ice Detection Using Delay-Doppler Maps: First Results from the U.K. TechDemoSat-1 Mission. IEEE J. Sel. Top. Appl. Earth Obs. Remote Sens. 2016, 9, 4795-4801. [CrossRef] 
14. Yan, Q.; Huang, W.; Moloney, C. Neural Networks Based Sea Ice Detection and Concentration Retrieval from GNSS-R Delay-Doppler Maps. IEEE J. Sel. Top. Appl. Earth Obs. Remote Sens. 2017, 10, 3789-3798. [CrossRef]

15. Alonso-Arroyo, A.; Zavorotny, V.U.; Camps, A. Sea Ice Detection Using U.K. TDS-1 GNSS-R Data. IEEE Trans. Geosci. Remote Sens. 2017, 55, 4989-5001. [CrossRef]

16. Yan, Q.; Huang, W. Sea Ice Sensing from GNSS-R Data Using Convolutional Neural Networks. IEEE Geosci. Remote Sens. Lett. 2018, 15, 1510-1514. [CrossRef]

17. Li, W.; Cardellach, E.; Fabra, F.; Rius, A.; Ribó, S.; Martín-Neira, M. First spaceborne phase altimetry over sea ice using TechDemoSat-1 GNSS-R signals. Geophys. Res. Lett. 2017, 44, 8369-8376. [CrossRef]

18. Rodriguez-Alvarez, N.; Holt, B.; Jaruwatanadilok, S.; Podest, E.; Cavanaugh, K. An Arctic Sea Ice Multi-Step Classification Based on GNSS-R Data from the TDS-1 Mission. Remote Sens. Environ. 2019, 230, 111202. [CrossRef]

19. Clarizia, M.P.; Ruf, C.S. Wind Speed Retrieval Algorithm for the Cyclone Global Navigation Satellite System (CYGNSS) Mission. IEEE Trans. Geosci. Remote Sens. 2016, 54, 4419-4432. [CrossRef]

20. Crespo, J.A.; Posselt, D.J.; Naud, C.M.; Bussy-Virat, C. Assessing CYGNSS's Potential to Observe Extratropical Fronts and Cyclones. J. Appl. Meteorol. Climatol. 2017, 56, 2027-2034. [CrossRef]

21. Morris, M.; Ruf, C.S. Estimating Tropical Cyclone Integrated Kinetic Energy with the CYGNSS Satellite Constellation. J. Appl. Meteorol. Climatol. 2017, 56, 235-245. [CrossRef]

22. Rodriguez-Alvarez, N.; Garrison, L.G. Generalized Linear Observables for Ocean Wind Retrieval from Calibrated GNSS-R Delay-Doppler Maps. IEEE Trans. Geosci. Remote Sens. 2016, 54, 1142-1155. [CrossRef]

23. Chew, C.C.; Small, E.E. Soil moisture sensing using spaceborne GNSS reflections: Comparison of CYGNSS reflectivity to SMAP soil moisture. Geophys. Res. Lett. 2018, 45, 4049-4405. [CrossRef]

24. Carreno-Luengo, H.; Luzi, G.; Crosetto, M. Sensitivity of CyGNSS Bistatic Reflectivity and SMAP Microwave Radiometry Brightness Temperature to Geophysical Parameters Over Land Surfaces. IEEE J. Sel. Top. Appl. Earth Obs. Remote Sens. 2018, 12, 107-122. [CrossRef]

25. Jensen, K.; McDonald, K.; Podest, E.; Rodriguez-Alvarez, N.; Horna, V.; Steiner, N. Assessing L-band GNSS-reflectometry and imaging radar for detecting sub-canopy inundation dynamics in a tropical wetlands complex. Remote Sens. 2018, 10, 1431. [CrossRef]

26. Rodriguez-Alvarez, N.; Podest, E.; Jensen, K.; McDonald, K.C. Classifying Inundation in a Tropical Wetlands Complex with GNSS-R. Remote Sens. 2019, 11, 1053. [CrossRef]

27. Morris, M.; Chew, C.; Reager, J.T.; Shah, R.; Zuffada, C. A Novel Approach to Monitoring Wetland Dynamics using CYGNSS: Everglades Case Study. Remote Sens. Environ. 2019, 233, 111417. [CrossRef]

28. Chew, C.; Reager, J.T.; Small, E. CYGNSS data map flood inundation during the 2017 Atlantic hurricane season. Sci. Rep. 2018, 8, 9336. [CrossRef]

29. Wan, W.; Liu, B.; Zeng, Z.; Chen, X.; Wu, G.; Xu, L.; Chen, X.; Hong, Y. Using CYGNSS data to monitor China's flood inundation during typhoon and extreme precipitation events in 2017. Remote Sens. 2019, 11, 854. [CrossRef]

30. Chew, C.; Lowe, S.; Parazoo, N.; Esterhuizen, S.; Oveisgharan, S.; Podest, E.; Zuffada, C.; Freedman, A. SMAP radar receiver measures land surface freeze/thaw state through capture of forward-scattered L-band signals. Remote Sens. Environ. 2017, 198, 333-344. [CrossRef]

31. Carreno-Luengo, H.; Lowe, S.; Zuffada, C.; Esterhuizen, S.; Oveisgharan, S. Spaceborne GNSS-R from the SMAP Mission: First Assessment of Polarimetric Scatterometry over Land and Cryosphere. Remote Sens. 2017, 9, 362. [CrossRef]

32. Rodriguez-Alvarez, N.; Podest, E. Characterization of the Land Freeze/Thaw State with SMAP-Reflectometry. In Proceedings of the IEEE International Geoscience and Remote Sensing Symposium 2019, Yokohama, Japan, 28 July-2 August 2019.

33. Rodriguez-Alvarez, N.; Misra, S.; Morris, M. Sensitivity Analysis of SMAP-Reflectometry (SMAP-R) Signals to Vegetation Water Content. In Proceedings of the IEEE International Geoscience and Remote Sensing Symposium 2019, Yokohama, Japan, 28 July-2 August 2019.

34. Ulaby, F.T.; Long, D.G. (Eds.) Microwave Radar and Radiometric Remote Sensing; University of Michigan Press: Ann Arbor, MI, USA, 2014.

35. Zavorotny, V.U.; Voronovich, A.G. Scattering of GPS signals from the ocean with wind remote sensing application. IEEE Trans. Geosci. Remote Sens. 2000, 38, 951-964. [CrossRef] 
36. Voronovich, A.G.; Zavorotny, V.U. Bistatic radar equation for signals of opportunity revisited. IEEE Trans. Geosci. Remote Sens. 2018, 56, 1959-1968. [CrossRef]

37. Clarizia, M.P.; Ruf, C.S. On the Spatial Resolution of GNSS Reflectometry. IEEE Geosci. Remote Sens. Lett. 2016, 13, 1064-1068. [CrossRef]

38. Camps, A. Spatial Resolution in GNSS-R Under Coherent Scattering. IEEE Geosci. Remote Sens. Lett 2019. (Early Access). [CrossRef]

39. Park, J.; Johnson, J.T.; O'Brien, A.; Lowe, S.T. An Examination of TDS-1 GNSS-R Returns over Land Surfaces. In Proceedings of the USNC-URSI NRSM, Boulder, CO, USA, 6-9 January 2016.

40. Park, J.; Johnson, J.T.; O'Brien, A. TDS-1 Coherent Returns over Sea Ice and Land Surfaces. In Proceedings of the GNSS+R 2017 Conference, Ann Arbor, MI, USA, 23-25 May 2017; Available online: http://www.gnssr2017.org/images/Thursday_afternoon/GNSS+R2017_TH_PM_7_Park_ TDS1_Coherent_Returns_over_Sea_Ice_and_Land_Surfaces.pdf (accessed on 3 June 2019).

41. Steigenberger, P.; Thölert, S.; Montenbruck, O. Flex power on GPS Block IIR-M and IIF. GPS Solut. 2019, 23, 8. [CrossRef]

42. Peng, J.; Misra, S.; Piepmeier, J.R.; Dinnat, E.P.; Yueh, S.H.; Meissner, T.; Le Vine, D.M.; Shelton, K.E.; Freedman, A.P.; Dunbar, R.S.; et al. Soil Moisture Active/Passive (SMAP) L-Band Microwave Radiometer Post-Launch Calibration Upgrade. IEEE J. Sel. Top. Appl. Earth Obs. Remote Sens. 2018, 12, 1647-1657. [CrossRef]

43. NSIDC: National Snow and Ice Data Center, University of Boulder, Colorado. Available online: https: //nsidc.org (accessed on 1 October 2019).

44. Fetterer, F.; Knowles, K.; Meier, W.N.; Savoie, M.; Windnagel, A.K. Sea Ice Index; Version 3; NSIDC: National Snow and Ice Data Center: Boulder, CO, USA, 2017. [CrossRef]

45. Amelung, F.; Forster, R.; Misra, T.; Bawden, G.; Glasscoe, M.; Monaldo, F.; Borsa, A.; Hager, B.; Oza, S.; Buckley, S.; et al. Nasa-ISRO Synthetic Aperture Radar (NISAR) Handbook. Available online: https: //nisar.jpl.nasa.gov/files/nisar/NISAR_Science_Users_Handbook1.p (accessed on 1 October 2019).

(C) 2019 by the authors. Licensee MDPI, Basel, Switzerland. This article is an open access article distributed under the terms and conditions of the Creative Commons Attribution (CC BY) license (http://creativecommons.org/licenses/by/4.0/). 